\title{
NUMERICAL STUDY OF A QUANTUM-DIFFUSIVE SPIN MODEL FOR TWO-DIMENSIONAL ELECTRON GASES*
}

\author{
LUIGI BARLETTI ${ }^{\dagger}$, FLORIAN MÉHATS ${ }^{\ddagger}$, CLAUDIA NEGULESCU ${ }^{\S}$, AND STEFAN \\ POSSANNER
}

\begin{abstract}
We investigate the time evolution of spin densities in a two-dimensional electron gas subjected to Rashba spin-orbit coupling on the basis of the quantum drift-diffusive model derived in [L. Barletti and F. Méhats, J. Math. Phys., 51, 053304(20), 2010]. This model assumes that the electrons are in a quantum equilibrium state in the form of a Maxwellian operator. The resulting quantum drift-diffusion equations for spin-up and spin-down densities are coupled in a non-local manner via two spin chemical potentials (Lagrange multipliers) and via off-diagonal elements of the equilibrium spin density and spin current matrices, respectively. We present two space-time discretizations of the model, one semi-implicit and one explicit, which also comprise the Poisson equation in order to account for electron-electron interactions. In a first step, pure time discretization is applied in order to prove the well-posedness of the two schemes, both of which are based on a functional formalism to treat the non-local relations between spin densities. We then use the fully space-time discrete schemes to simulate the time evolution of a Rashba electron gas confined in a bounded domain and subjected to spin-dependent external potentials. Finite difference approximations are first order in time and second order in space. The discrete functionals introduced are minimized with the help of a conjugate gradientbased algorithm where the Newton method is applied in order to find the respective line minima. The numerical convergence in the long-time limit of a Gaussian initial condition towards the solution of the corresponding stationary Schrödinger-Poisson problem is demonstrated for different values of the parameters $\varepsilon$ (semiclassical parameter), $\alpha$ (Rashba coupling parameter), $\Delta x$ (grid spacing), and $\Delta t$ (time step). Moreover, the performances of the semi-implicit and the explicit scheme are compared.
\end{abstract}

Key words. Rashba spin-orbit coupling, spin relaxation, entropic quantum drift-diffusion, quantum Liouville, Schrödinger-Poisson drift-diffusion, spin diffusion.

AMS subject classifications. $\quad 65 \mathrm{~K} 10,65 \mathrm{M} 12,65 \mathrm{~N} 25,76 \mathrm{Y} 05,82 \mathrm{C} 10,82 \mathrm{D} 37,81 \mathrm{R} 25$.

\section{Introduction}

The purpose of this paper is the numerical study of the quantum diffusive model for a spin-orbit system introduced in [2] with the aim of developing numerical tools for the investigation of spin-based electronic devices.

Diffusive models offer a simple, yet fairly accurate, description of charge transport, and, for this reason, they have a long-standing tradition in semiconductor modeling. Classical drift-diffusion equations for semiconductors [12] were first derived by van Roosbroeck [19] while Poupaud [18] proved their rigorous derivation from the Boltzmann equation. Quantum-corrected drift-diffusion equations were proposed in $[1,7]$ and were later derived by using a quantum version of the maximum entropy principle by Degond, Méhats, and Ringhofer [5, 6]. Finally, fully-quantum diffusive equations, still based on the quantum maximum entropy principle, were proposed in $[4,5]$. In view of recent progresses in controlling the electron spin, it is highly desirable to extend the

*Received: January 9, 2013; accepted (in revised form): July 5, 2014. Communicated by Lorenzo Pareschi.

${ }^{\dagger}$ Dipartimento di Matematica, Università di Firenze, Viale Morgagni, 67/a - 50134, Florence, Italia (barletti@math.unifi.it).

${ }^{\ddagger}$ INRIA IPSO Team, IRMAR, Université de Rennes 1, Campus de Beaulieu 35042 Cedex, Rennes, France (florian.mehats@univ-rennes1.fr).

§IMT, Université Paul Sabatier, 118 Route de Narbonne, 31400, Toulouse, France (claudia.negulescu@math.univ-toulouse.fr).

๑IMT, Université Paul Sabatier, 118 Route de Narbonne, 31400, Toulouse, France (Stefan.Possanner@math.univ-toulouse.fr). 
drift-diffusion description to the spinorial case. The existing semiclassical drift-diffusion models for spin systems can be classified into two categories: the two-component models [9] and the spin-polarized, or matrix, models [9, 17, 20]. Both models have been used in practice; however, their mathematical derivation is still at the very beginning.

As far as we know, a fully-quantum diffusive model of a spin system was first reported in [2] where a two-component diffusive model for a 2-dimensional electrons gas with spin-orbit interaction was derived. Such a model, which will be considered from the numerical point of view in the present work, is based on the quantum maximum entropy principle and concerns electrons with a spin-orbit Hamiltonian of Rashba type $[3]:$

$$
H=\left(\begin{array}{cc}
-\frac{\hbar^{2}}{2} \Delta+V & \alpha \hbar\left(\partial_{x}-i \partial_{y}\right) \\
-\alpha \hbar\left(\partial_{x}+i \partial_{y}\right) & -\frac{\hbar^{2}}{2} \Delta+V
\end{array}\right)
$$

Here, $(x, y)$ are the spatial coordinates of the 2-dimensional region where the electrons are assumed to be confined, $\alpha$ is the Rashba constant, and $V$ is a potential term which may consist of an "external" part (representing e.g. a gate or an applied potential) and a self-consistent part, accounting for Coulomb interactions in the mean-field approximation.

The Rashba effect $[3,21]$ is a spin-orbit interaction undergone by electrons that are confined in an asymmetric 2-dimensional well (here, perpendicular to the $z$ direction). Due to this interaction, the spin vector has a precession around a direction in the plane $(x, y)$ perpendicular to the electron momentum $p=\left(p_{x}, p_{y}\right)$, the precession speed being $\alpha|p|$. Since it does not involve built-in magnetic fields, and hence may be implemented by means of standard silicon technologies, the Rashba effect is expected to be a key ingredient for the realization of the so-called S-FET (Spin Field Effect Transistor) [21], a "spintronic" device in which the information is carried by the electron spin rather than by the electronic current (as in the usual electronic devices). This may lead to electronic devices of higher speed and lower power consumption. The purpose of this work is to contribute to the understanding of how the Rashba effect can be employed in order to control the spin transport in these devices.

Let us summarize briefly the derivation of the here investigated quantum diffusive model. The starting point is the von Neumann equation (i.e. the Schrödinger equation for mixed states) for the Hamiltonian (1.1) endowed with a collisional term of BGK type

$$
i \hbar \partial_{t} \varrho(t)=[H, \varrho(t)]+\frac{i}{\tau}\left(\varrho_{e q}-\varrho(t)\right),
$$

where $\varrho(t)=\left(\varrho_{i j}(t)\right)$ is the $2 \times 2$ density operator, representing the time-dependent mixed state of the system, and $\tau$ is the relaxation time. According to the theory developed in $[6,5]$, the local equilibrium state $\varrho_{e q}$ is chosen as the maximizer of a free energylike functional subject to the constraint of sharing with $\varrho(t)$ the local moments we are interested in, here the spin-up and spin-down (with respect to the $z$ direction) electron densities $n_{1}, n_{2}$ (or, equivalently, the total electronic density $n_{1}+n_{2}$ and the polarization $\left.n_{1}-n_{2}\right)$. Then, the maximizer, which has the form of a Maxwellian operator, contains as many Lagrange multipliers (chemical potentials) as the chosen moments. These multipliers furnish the degrees of freedom necessary to satisfy the constraint equations. In our case, therefore, the local equilibrium state contains two chemical potentials, $A_{1}$ and $A_{2}$, which depend on $n_{1}$ and $n_{2}$ through the constraint equations. The rigorous proof of realizability of the quantum Maxwellian associated to a given density and 
current was obtained in $[13,14]$ for a scalar (i.e. non spinorial) Hamiltonian. By assuming $\tau \ll 1$ and applying the Chapman-Enskog method, the von Neumann equation leads in the limit to the "quantum drift-diffusive" system (2.1) for the unknown densities $n_{1}$ and $n_{2}$. Apart from the chemical potentials $A_{1}$ and $A_{2}$, which depend on $n_{1}$ and $n_{2}$ through the constraint, the system also contains some extra moments, namely the off-diagonal density $n_{21}$ and currents $J_{21}^{x}, J_{21}^{y}$ which are computed via the equilibrium state and which depend on $n_{1}$ and $n_{2}$ as well. Note that, with respect to the original Hamiltonian (1.1), we shall work with a scaled version (see the Hamiltonian (2.4) which also contains the chemical potentials) in which $\varepsilon$ is the scaled Planck constant and $\alpha$ is rescaled as $\varepsilon \alpha$. This is, therefore, a semiclassical scaling with the additional assumption of small Rashba constant. Of course, the parameter $\varepsilon$ is unimportant as long as we are not interested in the semiclassical behavior but becomes relevant when we look for a semiclassical expansion of the model for small $\varepsilon$.

In summary, the diffusive Equation (2.1), equations (2.3)-(2.7), which represent the equilibrium state and the constraints, and the Poisson equation (2.2) for the selfconsistent potential, constitute the quantum diffusive model we are going to analyze numerically in this work. Needless to say, the model (2.1)-(2.7) is rather implicit and involved, and requires a very careful numerical treatment. The aim of the present paper is thus to present two discrete versions of (2.1)-(2.7) suitable for time-resolved simulation of the spin densities $n_{1}$ and $n_{2}$ in a spatially confined, two-dimensional electron gas. In both schemes, the finite-difference approximations of the occurring derivatives are first order in time and second order in space. At the core of the numerical study of the present model is the minimization of a functional that either maps from $\mathbb{R}^{3 P}$ to $\mathbb{R}$ (in the first scheme) or from $\mathbb{R}^{2 P}$ to $\mathbb{R}$ (in the second scheme) where $P$ is the number of points on the space grid. We present an algorithm that uses a combination of the conjugate gradient method and the Newton method in order to find the minimum of the respective functional at each time step.

The paper is organized as follows. In Section 2, the continuous model is introduced and is endowed with suitable initial and boundary conditions. In Section 3, we perform two different time discretizations of the continuous model and give a formal proof of the well-posedness of each of the two schemes. Then, in Section 4, two fully discrete schemes (i.e. both in time and space) are introduced and analyzed as well. Finally, Section 5 is devoted to numerical experiments. Details of the proofs and of the discretization matrices are deferred to the appendices.

\section{The quantum spin drift-diffusion model}

Let us start with the presentation of the quantum diffusive model introduced in [2]. The model describes the evolution of the spin-up and the spin-down densities $n_{1}$ and $n_{2}$, respectively, of a two-dimensional electron gas by means of the following quantum drift-diffusion equations:

$$
\begin{aligned}
\partial_{t} n_{1} & +\nabla \cdot\left(n_{1} \nabla\left(A_{1}-V_{s}\right)\right)+\alpha\left(A_{1}-A_{2}\right) \mathcal{R e}\left(\mathcal{D} n_{21}\right)-2 \alpha \mathcal{R e}\left(n_{21} \mathcal{D}\left(A_{2}-V_{s}\right)\right) \\
& -\frac{2 \alpha}{\varepsilon}\left(A_{1}-A_{2}\right) \mathcal{I} \mathrm{m}\left(J_{21}^{x}-i J_{21}^{y}\right)=0, \\
\partial_{t} n_{2} & +\nabla \cdot\left(n_{2} \nabla\left(A_{2}-V_{s}\right)\right)+\alpha\left(A_{1}-A_{2}\right) \mathcal{R e}\left(\mathcal{D} n_{21}\right)+2 \alpha \mathcal{R e}\left(n_{21} \mathcal{D}\left(A_{1}-V_{s}\right)\right) \\
& +\frac{2 \alpha}{\varepsilon}\left(A_{1}-A_{2}\right) \mathcal{I} \mathrm{m}\left(J_{21}^{x}-i J_{21}^{y}\right)=0 .
\end{aligned}
$$

Here, $\nabla=\left(\partial_{x}, \partial_{y}\right), \mathcal{D}=\partial_{x}-i \partial_{y}, A_{1}$, and $A_{2}$ denote the two Lagrange multipliers $\left(A_{1}-\right.$ $V_{s}$ and $A_{2}-V_{s}$ being the chemical potentials), $V_{s}$ stands for the self-consistent potential 
arising from the electron-electron interaction, and $n_{21}, J_{21}^{x}$, and $J_{21}^{y}$ are off-diagonal elements of the spin-density matrix $N$ and the spin-current tensor $J$ written in (2.6) and (2.7), respectively. The parameter $\alpha>0$ denotes the scaled Rashba constant and $\varepsilon>0$ stands for the scaled Planck constant (for details regarding the scaling we refer to $[2])$. The self-consistent potential $V_{s}$ is determined by the Poisson equation,

$$
-\gamma^{2} \Delta V_{s}=n_{1}+n_{2}
$$

where $\gamma>0$ is proportional to the occurring Debye length. The system (2.1)-(2.2) is closed through the fact that the electrons are assumed to be in a quantum local equilibrium state at all times. This constraint allows one to relate the Lagrange multipliers $A=\left(A_{1}, A_{2}\right)$ to the spin densities $n_{1}$ and $n_{2}$ as well as to the spin-mixing quantities $n_{21}$ and $J_{21}$, respectively. More precisely, if $H(A)$ denotes the system Hamiltonian, the equilibrium state operator is given by

$$
\varrho_{e q}=\exp (-H(A))
$$

where $\exp (\cdot)$ here denotes the operator exponential. In the present case, the Hamiltonian is given by

$$
\begin{gathered}
H(A): D(H) \subset\left(L^{2}(\Omega, \mathbb{C})\right)^{2} \rightarrow\left(L^{2}(\Omega, \mathbb{C})\right)^{2}, \quad D(H) \subset\left(H^{2}(\Omega, \mathbb{C})\right)^{2}, \\
H(A)=\left(\begin{array}{cc}
-\frac{\varepsilon^{2}}{2} \Delta+V_{\text {ext }, 1}+A_{1} & \varepsilon^{2} \alpha\left(\partial_{x}-i \partial_{y}\right) \\
-\varepsilon^{2} \alpha\left(\partial_{x}+i \partial_{y}\right) & -\frac{\varepsilon^{2}}{2} \Delta+V_{\text {ext }, 2}+A_{2}
\end{array}\right),
\end{gathered}
$$

where $\Omega \subset \mathbb{R}^{2}$ denotes the bounded domain where the electrons are assumed to be confined. In what follows, we shall denote $\mathbf{x}=(x, y) \in \Omega$. Hence, we have introduced two external, time-independent potentials $V_{\text {ext,1 }}(\mathbf{x})$ and $V_{\text {ext,2 }}(\mathbf{x})$ for the spin-up and the spin-down electrons, respectively. Assuming that $H(A)$ has a pure point spectrum, the eigenvalues and the eigenvectors of $H(A)$, denoted by $\lambda_{l}(A)$ and $\psi_{l}(A)=\left(\psi_{l}^{1}(A), \psi_{l}^{2}(A)\right)$, $l \in \mathbb{N}$, respectively, are solutions of

$$
H(A) \psi_{l}(A)=\lambda_{l}(A) \psi_{l}(A)
$$

and link the Lagrange multipliers to the spin-density matrix $N$ as well as to the spincurrent matrix $J$, according to

$$
\begin{aligned}
N & =\sum_{l} e^{-\lambda_{l}}\left(\begin{array}{cc}
\left|\psi_{l}^{1}\right|^{2} & \psi_{l}^{1} \overline{\psi_{l}^{2}} \\
\psi_{l}^{2} \overline{\psi_{l}^{1}}\left|\psi_{l}^{2}\right|^{2}
\end{array}\right)=\left(\begin{array}{cc}
n_{1} & \bar{n}_{21} \\
n_{21} & n_{2}
\end{array}\right) \\
J & =-\frac{i \varepsilon}{2} \sum_{l} e^{-\lambda_{l}}\left(\begin{array}{ll}
\overline{\psi_{l}^{1}} \nabla \psi_{l}^{1}-\psi_{l}^{1} \nabla \overline{\psi_{l}^{1}} & \overline{\psi_{l}^{2}} \nabla \psi_{l}^{1}-\psi_{l}^{1} \nabla \overline{\psi_{l}^{2}} \\
\overline{\psi_{l}^{1}} \nabla \psi_{l}^{2}-\psi_{l}^{2} \nabla \overline{\psi_{l}^{1}} & \overline{\psi_{l}^{2}} \nabla \psi_{l}^{2}-\psi_{l}^{2} \nabla \overline{\psi_{l}^{2}}
\end{array}\right) \\
& =\left(\begin{array}{cc}
J_{1} & \bar{J}_{21} \\
J_{21} & J_{2}
\end{array}\right) .
\end{aligned}
$$

The formulas (2.6) and (2.7) are the standard textbook expressions for the spin-density and the spin-current, respectively, corresponding to the density operator $(2.3)$. The system (2.1)-(2.2) is now closed through the non-local relations $N(A)$ and $J(A)$ given by equations (2.4)-(2.7). As we do not have a proof of the invertibility of these relations, 
in other words whether it is possible to compute $A\left(n_{1}, n_{2}\right)$, Equation (2.1) can also be viewed as evolution equations for the Lagrange multipliers $A_{1}$ and $A_{2}$ rather than for the spin densities $n_{1}$ and $n_{2}$. Indeed, the two time-discretizations of the system (2.1)-(2.7), which will be developed in Section 3, represent these two possible viewpoints regarding the evolution Equation (2.1).

Let us now come to the boundary conditions of our problem. The considered spatial domain $\Omega \subset \mathbb{R}^{2}$ is assumed to be bounded with regular boundary $\partial \Omega$. We shall impose Dirichlet boundary conditions for the eigenvectors $\psi_{l}$,

$$
\psi_{l}(\mathbf{x})=0 \quad \text { for } \quad \mathbf{x} \in \partial \Omega
$$

hence the current across the domain boundary $\partial \Omega$ is zero. As we will briefly show at the end of this section, the Hamiltonian $(2.4)$ is not hermitian in $\left(L^{2}(\Omega, \mathbb{C})\right)^{2}$ when imposing Neumann boundary conditions. The study of this problem, as well as the implementation of transparent boundary conditions, can be matter for a future work. The self-consistent potential $V_{s}$ is supplemented with Dirichlet conditions too:

$$
V_{s}(\mathbf{x})=0 \quad \text { for } \quad \mathbf{x} \in \partial \Omega .
$$

The Lagrange multipliers $A_{1}$ and $A_{2}$ are allowed to vary freely at the boundary. Therefore, we take Neumann conditions,

$$
\begin{array}{lll}
\nabla\left(A_{1}(\mathbf{x})-V_{s}(\mathbf{x})\right) \cdot \nu(\mathbf{x})=0 & \text { for } & \mathbf{x} \in \partial \Omega \\
\nabla\left(A_{2}(\mathbf{x})-V_{s}(\mathbf{x})\right) \cdot \nu(\mathbf{x})=0 & \text { for } & \mathbf{x} \in \partial \Omega .
\end{array}
$$

Here, $\nu(\mathbf{x})$ denotes the outward normal to the boundary $\partial \Omega$ at $\mathbf{x}$. As far as initial conditions are concerned, one has two choices depending on the point of view of the evolution Equation (2.1). Since we do not know whether or not (2.6) is invertible, the safe approach is to provide initial data for the chemical potentials. However, from the viewpoint of device modeling, it is more appealing to start from initial spin densities. We shall take the latter approach and assume that $n_{1}(0, \mathbf{x})$ and $n_{2}(0, \mathbf{x})$ are smooth and bounded.

In summary, we have the following quantum spin-drift-diffusion model:

$$
\begin{aligned}
& \partial_{t} n_{1}+\nabla \cdot\left(n_{1} \nabla\left(A_{1}-V_{s}\right)\right)+\alpha\left(A_{1}-A_{2}\right) \mathcal{R e}\left(\mathcal{D} n_{21}\right) \\
& \quad-2 \alpha \mathcal{R e}\left(n_{21} \mathcal{D}\left(A_{2}-V_{s}\right)\right)-\frac{2 \alpha}{\varepsilon}\left(A_{1}-A_{2}\right) \mathcal{I} \mathrm{m}\left(J_{21}^{x}-i J_{21}^{y}\right)=0, \\
& \partial_{t} n_{2}+\nabla \cdot\left(n_{2} \nabla\left(A_{2}-V_{s}\right)\right)+\alpha\left(A_{1}-A_{2}\right) \mathcal{R e}\left(\mathcal{D} n_{21}\right) \\
& \quad+2 \alpha \mathcal{R} \mathrm{e}\left(n_{21} \mathcal{D}\left(A_{1}-V_{s}\right)\right)+\frac{2 \alpha}{\varepsilon}\left(A_{1}-A_{2}\right) \mathcal{I} \mathrm{m}\left(J_{21}^{x}-i J_{21}^{y}\right)=0, \\
& -\gamma^{2} \Delta V_{s}=n_{1}+n_{2}, \\
& H(A) \psi_{l}(A)=\lambda_{l}(A) \psi_{l}(A), \\
& N=\sum_{l} e^{-\lambda_{l}(A)}\left(\begin{array}{cc}
\left|\psi_{l}^{1}(A)\right|^{2} & \psi_{l}^{1}(A) \overline{\psi_{l}^{2}}(A) \\
\psi_{l}^{2}(A) \overline{\psi_{l}^{1}}(A) & \left|\psi_{l}^{2}(A)\right|^{2}
\end{array}\right),
\end{aligned}
$$


$J_{21}=-\frac{i \varepsilon}{2} \sum_{l} e^{-\lambda_{l}(A)}\left(\overline{\psi_{l}^{1}}(A) \nabla \psi_{l}^{2}(A)-\psi_{l}^{2}(A) \nabla \overline{\psi_{l}^{1}}(A)\right)$

The Hamiltonian $H(A)$ is given by (2.4) and the problem is supplemented with the following initial and boundary conditions:

$$
\begin{aligned}
& n_{1}(t=0, \mathbf{x})=n_{1}^{0}(\mathbf{x}), \quad n_{2}(t=0, \mathbf{x})=n_{2}^{0}(\mathbf{x}) \text { for } \quad \mathbf{x} \in \Omega, \\
& V_{s}(\mathbf{x})=0 \text { for } \mathbf{x} \in \partial \Omega \\
& \psi_{l}(\mathbf{x})=0 \text { for } \quad \mathbf{x} \in \partial \Omega, \\
& \nabla\left(A_{1}(\mathbf{x})-V_{s}(\mathbf{x})\right) \cdot \nu(\mathbf{x})=0 \text { for } \quad \mathbf{x} \in \partial \Omega \\
& \nabla\left(A_{2}(\mathbf{x})-V_{s}(\mathbf{x})\right) \cdot \nu(\mathbf{x})=0 \text { for } \quad \mathbf{x} \in \partial \Omega
\end{aligned}
$$

Let us finish this section by remarking that the Hamiltonian (2.4) is not hermitian in $\left(L^{2}(\Omega, \mathbb{C})\right)^{2}$ for Neumann boundary conditions. Indeed, let us consider

$$
(\chi, H(A) \psi)_{L^{2}}=\int_{\Omega}\left(\overline{\chi^{1}}, \overline{\chi^{2}}\right)\left(\begin{array}{c}
-\frac{\varepsilon^{2}}{2} \Delta \psi^{1}+\left(V_{e x t, 1}+A_{1}\right) \psi^{1}+\varepsilon^{2} \alpha\left(\partial_{x}-i \partial_{y}\right) \psi^{2} \\
-\frac{\varepsilon^{2}}{2} \Delta \psi^{2}+\left(V_{e x t, 2}+A_{2}\right) \psi^{2}-\varepsilon^{2} \alpha\left(\partial_{x}+i \partial_{y}\right) \psi^{1}
\end{array}\right) d \mathbf{x}
$$

where $(\cdot, \cdot)_{L^{2}}$ denotes the scalar product in $\left(L^{2}(\Omega, \mathbb{C})\right)^{2}$. Specifically, let us look at the Rashba coupling terms,

$$
\begin{aligned}
& \int_{\Omega}\left(\overline{\chi^{1}}\left(\partial_{x}-i \partial_{y}\right) \psi^{2}-\overline{\chi^{2}}\left(\partial_{x}+i \partial_{y}\right) \psi^{1}\right) d \mathbf{x} \\
= & \left.-\int_{\Omega} \psi^{2}\left(\partial_{x}-i \partial_{y}\right) \overline{\chi^{1}} d \mathbf{x}+\int_{\Omega} \psi^{1}\left(\partial_{x}+i \partial_{y}\right) \overline{\chi^{2}}\right) d \mathbf{x} \\
& +\int_{\partial \Omega} \overline{\chi^{1}} \psi^{2}(1,-i) \cdot \nu(\mathbf{x}) d \sigma-\int_{\partial \Omega} \overline{\chi^{2}} \psi^{1}(1, i) \cdot \nu(\mathbf{x}) d \sigma .
\end{aligned}
$$

Here, the boundary terms do not vanish when imposing Neumann conditions. However, if we considered the problem in the whole space $\Omega=\mathbb{R}^{2}$, the boundary terms would vanish and the Hamiltonian would be hermitian. Considering the problem in the whole $\mathbb{R}^{2}$ means, from the numerical point of view, imposing transparent boundary conditions for $\psi$.

\section{Semi-discretization in time}

In this section, we make a first step towards a full space-time discretization of the system (2.8)-(2.14) by discretizing the time domain. The purpose of the semidiscretization is two-fold. Firstly, since the space discretization of the present twodimensional spin model is quite involved, the functional formalism which will be applied in this work becomes more transparent in the semi-discrete case than in the fully discrete case. Secondly, in contrast to the continuous case (2.8)-(2.14), existence and uniqueness of solutions of the semi-discrete system can be proven. Two different semidiscretizations will be presented. The first one was studied in [8] for a scalar quantum diffusive model (without the Rashba spin-orbit coupling). We shall use some of the techniques elaborated in [8] and apply them to the present spin model. The second semi-discrete scheme is an explicit one which relies heavily on the ability to invert the relation (2.12). Its benefits lie in the fact that, when passing to the full discretization, its treatment is far less involved as compared to the first scheme. 
In the subsequent analysis, the identities

$$
\begin{aligned}
& \left(A_{1}-A_{2}\right) \mathcal{D} n_{21}^{k}-2 n_{21}^{k} \mathcal{D}\left(A_{2}\right)=\mathcal{D}\left(n_{21}^{k}\left(A_{1}-A_{2}\right)\right)-n_{21}^{k} \mathcal{D}\left(A_{1}+A_{2}\right), \\
& \left(A_{1}-A_{2}\right) \mathcal{D} n_{21}^{k}+2 n_{21}^{k} \mathcal{D}\left(A_{1}\right)=\mathcal{D}\left(n_{21}^{k}\left(A_{1}-A_{2}\right)\right)+n_{21}^{k} \mathcal{D}\left(A_{1}+A_{2}\right)
\end{aligned}
$$

will be helpful.

3.1. A first semi-discrete system. Suppose $T>0$ and let us discretize the temporal interval $[0, T]$ in the following homogeneous way:

$$
t_{k}=k \Delta t, \quad k \in\{0,1, \ldots, K\}, \quad \Delta t:=\frac{T}{K} .
$$

Then, inspired by [8], we choose the following time-discretization of the continuous problem (2.8)-(2.13):

$$
\begin{aligned}
& \frac{n_{1}\left(A^{k+1}\right)-n_{1}^{k}}{\Delta t}+\nabla \cdot\left(n_{1}^{k} \nabla\left(A_{1}^{k+1}-V_{s}^{k+1}\right)\right)+\alpha \mathcal{R e}\left[\mathcal{D}\left(n_{21}^{k}\left(A_{1}^{k+1}-A_{2}^{k+1}\right)\right)\right] \\
& -\alpha \mathcal{R e}\left[n_{21}^{k} \mathcal{D}\left(A_{1}^{k+1}+A_{2}^{k+1}-2 V_{s}^{k+1}\right)\right] \\
& -\frac{2 \alpha}{\varepsilon}\left(A_{1}^{k+1}-A_{2}^{k+1}\right) \mathcal{I} \mathrm{m}\left(J_{x}^{21, k}-i J_{y}^{21, k}\right)=0 \text {, } \\
& \frac{n_{2}\left(A^{k+1}\right)-n_{2}^{k}}{\Delta t}+\nabla \cdot\left(n_{2}^{k} \nabla\left(A_{2}^{k+1}-V_{s}^{k+1}\right)\right)+\alpha \mathcal{R} \mathrm{e}\left[\mathcal{D}\left(n_{21}^{k}\left(A_{1}^{k+1}-A_{2}^{k+1}\right)\right)\right] \\
& +\alpha \mathcal{R e}\left[n_{21}^{k} \mathcal{D}\left(A_{1}^{k+1}+A_{2}^{k+1}-2 V_{s}^{k+1}\right)\right] \\
& +\frac{2 \alpha}{\varepsilon}\left(A_{1}^{k+1}-A_{2}^{k+1}\right) \mathcal{I} \mathrm{m}\left(J_{x}^{21, k}-i J_{y}^{21, k}\right)=0, \\
& -\gamma^{2} \Delta V_{s}^{k+1}=n_{1}\left(A^{k+1}\right)+n_{2}\left(A^{k+1}\right), \\
& H\left(A^{k+1}\right) \psi_{l}^{k+1}=\lambda_{l}^{k+1} \psi_{l}^{k+1}, \\
& n_{1}\left(A^{k+1}\right)=\sum_{l} e^{-\lambda_{l}^{k+1}}\left|\psi_{l}^{1, k+1}\right|^{2}, \quad n_{2}\left(A^{k+1}\right)=\sum_{l} e^{-\lambda_{l}^{k+1}}\left|\psi_{l}^{2, k+1}\right|^{2} .
\end{aligned}
$$

In this scheme, one searches for the unknowns $\left(A^{k+1}, V_{s}^{k+1}\right)$ given $\left(N^{k}, J_{21}^{k}\right)$. The main difficulties concerning the solution of this system are the non-local relations (3.5)-(3.6). We shall thus construct a mapping $\left(A, V_{s}\right) \in\left(H^{1}(\Omega, \mathbb{R})\right)^{3} \mapsto \mathcal{F}\left(A, V_{s}\right) \in \mathbb{R}$ whose unique minimum $\left(A^{k+1}, V_{s}^{k+1}\right)$ is the solution of system (3.2)-(3.6). Once $A^{k+1}$ and the eigenvalues $\lambda_{l}^{k+1}$, respectively eigenvectors $\psi_{l}^{k+1}$, are known, equations (2.12)-(2.13) can be used to compute $\left(N^{k+1}, J_{21}^{k+1}\right)$ and the process can be repeated. Let us thus introduce the two functionals

$$
\mathcal{G}:\left(L^{2}(\Omega, \mathbb{R})\right)^{2} \rightarrow \mathbb{R}, \quad \mathcal{F}:\left(H^{1}(\Omega, \mathbb{R})\right)^{3} \rightarrow \mathbb{R}
$$

defined by

$$
\mathcal{G}(A):=\sum_{l} e^{-\lambda_{l}(A)}, \quad A \in\left(L^{2}(\Omega, \mathbb{R})\right)^{2}
$$


where $\lambda_{l}(A)$ are the eigenvalues of the Hamiltonian (2.4), and

$$
\mathcal{F}\left(A, V_{s}\right)=\mathcal{G}(A)+\mathcal{F}_{1}\left(A, V_{s}\right)+\mathcal{F}_{2}\left(A, V_{s}\right)+\mathcal{F}_{3}\left(A, V_{s}\right)+\mathcal{F}_{4}(A),
$$

where

$$
\begin{aligned}
& \mathcal{F}_{1}\left(A, V_{s}\right):=\frac{\Delta t}{2} \int_{\Omega} n_{1}^{k}\left|\nabla\left(A_{1}-V_{s}\right)\right|^{2} d \mathbf{x}+\frac{\Delta t}{2} \int_{\Omega} n_{2}^{k}\left|\nabla\left(A_{2}-V_{s}\right)\right|^{2} d \mathbf{x}, \\
& \mathcal{F}_{2}\left(A, V_{s}\right):=\frac{\gamma^{2}}{2} \int_{\Omega}\left|\nabla V_{s}\right|^{2} d \mathbf{x}+\int_{\Omega} n_{1}^{k}\left(A_{1}-V_{s}\right) d \mathbf{x}+\int_{\Omega} n_{2}^{k}\left(A_{2}-V_{s}\right) d \mathbf{x}, \\
& \mathcal{F}_{3}\left(A, V_{s}\right):=\alpha \Delta t \mathcal{R} \operatorname{e}\left\{\int_{\Omega} n_{21}^{k}\left(A_{1}-A_{2}\right) \mathcal{D}\left(A_{1}+A_{2}-2 V_{s}\right) d \mathbf{x}\right\}, \\
& \mathcal{F}_{4}(A):=\frac{\alpha \Delta t}{\varepsilon} \mathcal{I} \operatorname{m}\left\{\int_{\Omega}\left(A_{1}-A_{2}\right)^{2}\left(J_{x}^{21, k}-i J_{y}^{21, k}\right) d \mathbf{x}\right\} .
\end{aligned}
$$

The computation of the first and second Gateaux derivatives of the functionals (3.7)(3.12) can be found in Appendix B and C, respectively. One can immediately see that a solution $\left(A^{k+1}, V_{s}^{k+1}\right)$ of the semi-discrete system (3.2)-(3.6) satisfies

$$
\mathrm{d} \mathcal{F}\left(A^{k+1}, V_{s}^{k+1}\right)\left(\delta A, \delta V_{s}\right)=0, \quad \forall\left(\delta A, \delta V_{s}\right) \in\left(H^{1}(\Omega, \mathbb{R})\right)^{3},
$$

and inversely. Thus, it remains to show that $\mathcal{F}$ has a unique extremum (minimum). This can be achieved in two steps as detailed in Appendix C. First we show that, under suitable assumptions, the functional $\mathcal{F}$ is strictly convex. Then it is sufficient to show that $\mathcal{F}$ is coercive to obtain the existence and uniqueness of the extremum $\left(A^{k+1}, V_{s}^{k+1}\right)$, a solution of the system (3.2)-(3.6) (see Appendix C).

3.2. A second semi-discrete system. We suggest here an alternative way to discretize in time the quantum drift-diffusion model (2.8)-(2.14). It is based on the point of view that one advances the spin densities in time rather than the chemical potentials. We shall implement a forward Euler scheme:

$$
\begin{aligned}
& \frac{n_{1}^{k+1}-n_{1}^{k}}{\Delta t}+\nabla \cdot\left(n_{1}^{k} \nabla\left(A_{1}^{k}-V_{s}^{k}\right)\right)+\alpha \mathcal{R} \operatorname{e}\left\{\mathcal{D}\left(n_{21}^{k}\left(A_{1}^{k}-A_{2}^{k}\right)\right)\right\} \\
& -\alpha \mathcal{R e}\left(n_{21}^{k} \mathcal{D}\left(A_{1}^{k}+A_{2}^{k}-2 V_{s}^{k}\right)\right)-\frac{2 \alpha}{\varepsilon}\left(A_{1}^{k}-A_{2}^{k}\right) \mathcal{I} \mathrm{m}\left(J_{21}^{x, k}-i J_{21}^{y, k}\right) \\
= & 0, \\
& \frac{n_{2}^{k+1}-n_{2}^{k}}{\Delta t}+\nabla \cdot\left(n_{2}^{k} \nabla\left(A_{2}^{k}-V_{s}^{k}\right)\right)+\alpha \mathcal{R e}\left\{\mathcal{D}\left[n_{21}^{k}\left(A_{1}^{k}-A_{2}^{k}\right)\right]\right\} \\
& +\alpha \mathcal{R e}\left[n_{21}^{k} \mathcal{D}\left(A_{1}^{k}+A_{2}^{k}-2 V_{s}^{k}\right)\right]+\frac{2 \alpha}{\varepsilon}\left(A_{1}^{k}-A_{2}^{k}\right) \mathcal{I} \operatorname{m}\left(J_{21}^{x, k}-i J_{21}^{y, k}\right) \\
= & 0,
\end{aligned}
$$




$$
\begin{gathered}
N=\sum_{l} e^{-\lambda_{l}^{k}}\left(\begin{array}{c}
\left|\psi_{l}^{1, k}\right|^{2} \psi_{l}^{1, k} \overline{\psi_{l}^{2, k}} \\
\psi_{l}^{2, k} \overline{\psi_{l}^{1, k}}\left|\psi_{l}^{2, k}\right|^{2}
\end{array}\right), \\
J_{21}^{k}=-\frac{i \varepsilon}{2} \sum_{l} e^{-\lambda_{l}^{k}}\left(\overline{\psi_{l}^{1, k}} \nabla \psi_{l}^{2, k}-\psi_{l}^{2, k} \nabla \overline{\psi_{l}^{1, k}}\right) .
\end{gathered}
$$

In this case, given the spin-densities $\left(n_{1}^{k}, n_{2}^{k}\right)$, one first uses the Poisson equation (3.15) to get $V_{s}^{k}$ then inverts the relations (3.16)-(3.17) in order to get the chemical potentials $\left(A_{1}^{k}, A_{2}^{k}\right)$. Finally, one advances in time using the drift-diffusion equations (3.13)-(3.14) in order to get the new spin densities $\left(n_{1}^{k+1}, n_{2}^{k+1}\right)$, and then one repeats the steps. The inversion of the non-local relation (3.16)-(3.17) can be achieved by minimizing the functional $\mathcal{G}_{n}:\left(L^{2}(\Omega, \mathbb{R})\right)^{2} \rightarrow \mathbb{R}$ defined by

$$
\mathcal{G}_{n}(A):=\mathcal{G}(A)+\int_{\Omega} n_{1}^{k} A_{1} d \mathbf{x}+\int_{\Omega} n_{2}^{k} A_{2} d \mathbf{x}
$$

Indeed, the first derivative of this functional reads

$$
\begin{aligned}
\mathrm{d} \mathcal{G}_{n}(A)(\delta A)= & -\sum_{l} e^{-\lambda_{l}(A)} \int_{\Omega}\left(\left|\psi_{l}^{1}(A)\right|^{2} \delta A_{1}+\left|\psi_{l}^{2}(A)\right|^{2} \delta A_{2}\right) d \mathbf{x} \\
& +\int_{\Omega} n_{1}^{k} \delta A_{1} d \mathbf{x}+\int_{\Omega} n_{2}^{k} \delta A_{2} d \mathbf{x},
\end{aligned}
$$

which clearly implies that its zeros are solutions of (3.16)-(3.17). As shown in Appendix $\mathrm{B}$, the functional $\mathcal{G}_{n}$ is strictly convex and coercive and hence admits a unique extremum.

REMARK 3.1. The two semi-discrete systems presented in this section conserve the total mass $\left(n_{1}+n_{2}\right)$ because of the particular choice of Dirichlet boundary conditions for the eigenvectors $\psi_{l}$ of the Hamiltonian (2.4). This can be obtained by integrating the sum of the semi-discrete drift-diffusion equations for $n_{1}$ and $n_{2}$, equations (3.2)-(3.3) or equations (3.13)-(3.14), respectively, over the domain $\Omega$. The remaining boundary term is of the form

$$
\int_{\partial \Omega} n_{21}\left(A_{1}-A_{2}\right)(1,-i) \cdot \nu(\mathbf{x}) d \sigma
$$

which does not vanish for Neumann boundary conditions. This is in accordance with the remark at the end of Section 2 where we showed that Neumann conditions for $\psi_{l}$ lead to a non-hermitian Hamiltonian $(2.4)$ in $\left(L^{2}(\Omega, \mathbb{C})\right)^{2}$.

\section{Fully discrete system}

This section is devoted to the full discretization of the continuous spin QDD model (2.8)-(2.14). The time discretization was done in the previous section; now we focus on the space discretization. Let $x \in \Omega=[0,1] \times[0,1]$ with the discretization

$$
\begin{gathered}
x_{i j}=((j-1) \Delta x,(i-1) \Delta y), \quad j \in\{1,2, \ldots, M\}, \quad i \in\{1,2, \ldots, N\}, \\
\Delta x:=\frac{1}{M-1}, \quad \Delta y:=\frac{1}{N-1} .
\end{gathered}
$$

For functions $f(x)$ on $\Omega$, we write $f\left(x_{i j}\right)=f_{i j}$. A function $f(x)$ that is subject to homogenous Dirichlet boundary conditions on $\partial \Omega$ satisfies

$$
f_{1 j}=f_{N j}=0 \quad \forall j \in\{1,2, \ldots, M\}, \quad f_{i 1}=f_{i M}=0 \quad \forall i \in\{1,2, \ldots, N\} .
$$


We introduce the following index transformation:

$$
(i, j) \mapsto p \quad \forall \quad i \in\{2, \ldots, N-1\}, j \in\{2, \ldots, M-1\}
$$

defined by

$$
p=(N-2)(j-2)+i-1, \quad p=1, \ldots, P, \quad P:=(N-2)(M-2) .
$$

For discrete functions $\left(f_{i j}\right)_{i, j=2}^{N-1, M-1}$ in $\Omega$, the following vector notation will be implemented:

$$
\hat{f}:=\left(f_{p}\right)_{p=1}^{P} \in \mathbb{C}^{P} .
$$

The corresponding Euclidean scalar product is denoted by

$$
(\hat{f}, \hat{g})_{P}=\Delta x \Delta y \sum_{p} f_{p} \bar{g}_{p}=\Delta x \Delta y \sum_{i=2}^{N-1} \sum_{j=2}^{M-1} f_{i j} \bar{g}_{i j} .
$$

4.1. A first fully discrete system (scheme 1). The discretization matrices $D_{x}^{ \pm}, D_{y}^{ \pm}, D_{x}, D_{y}, \tilde{D}_{x}, \tilde{D}_{y}$, and $\Delta_{\text {dir }}$ used in the following are defined in Appendix D. In view of the boundary conditions (2.14), we choose the following space discretization of the semi-discrete system (3.2)-(3.6):

$$
\begin{aligned}
& \frac{\hat{n}_{1}\left(\hat{A}_{1}^{k+1}, \hat{A}_{2}^{k+1}\right)-\hat{n}_{1}^{k}}{\Delta t}-\frac{2 \alpha}{\varepsilon}\left(\hat{A}_{1}^{k+1}-\hat{A}_{2}^{k+1}\right) \circ \mathcal{I} \mathrm{m}\left(\hat{J}_{x}^{21, k}-i \hat{J}_{y}^{21, k}\right) \\
& -\frac{1}{2}\left(D_{x}^{+}\right)^{T}\left[\hat{n}_{1}^{k} \circ D_{x}^{+}\left(\hat{A}_{1}^{k+1}-\hat{V}_{s}^{k+1}\right)\right]-\frac{1}{2}\left(D_{x}^{-}\right)^{T}\left[\hat{n}_{1}^{k} \circ D_{x}^{-}\left(\hat{A}_{1}^{k+1}-\hat{V}_{s}^{k+1}\right)\right] \\
& -\frac{1}{2}\left(D_{y}^{+}\right)^{T}\left[\hat{n}_{1}^{k} \circ D_{y}^{+}\left(\hat{A}_{1}^{k+1}-\hat{V}_{s}^{k+1}\right)\right]-\frac{1}{2}\left(D_{y}^{-}\right)^{T}\left[\hat{n}_{1}^{k} \circ D_{y}^{-}\left(\hat{A}_{1}^{k+1}-\hat{V}_{s}^{k+1}\right)\right] \\
& -\alpha \mathcal{R e}\left\{\widetilde{D}_{x}^{T}\left[\hat{n}_{21}^{k} \circ\left(\hat{A}_{1}^{k+1}-\hat{A}_{2}^{k+1}\right)\right]\right\}+\alpha \mathcal{R e}\left\{i \widetilde{D}_{y}^{T}\left[\hat{n}_{21}^{k} \circ\left(\hat{A}_{1}^{k+1}-\hat{A}_{2}^{k+1}\right)\right]\right\} \\
& -\alpha \mathcal{R} e\left\{\hat{n}_{21}^{k} \circ\left[\widetilde{D}_{x}\left(\hat{A}_{1}^{k+1}+\hat{A}_{2}^{k+1}-2 \hat{V}_{s}^{k+1}\right)\right]\right\} \\
& +\alpha \mathcal{R e}\left\{i \hat{n}_{21}^{k} \circ\left[\widetilde{D}_{y}\left(\hat{A}_{1}^{k+1}+\hat{A}_{2}^{k+1}-2 \hat{V}_{s}^{k+1}\right)\right]\right\}=0, \\
& \frac{\hat{n}_{2}\left(\hat{A}_{1}^{k+1}, \hat{A}_{2}^{k+1}\right)-\hat{n}_{2}^{k}}{\Delta t}+\frac{2 \alpha}{\varepsilon}\left(\hat{A}_{1}^{k+1}-\hat{A}_{2}^{k+1}\right) \circ \mathcal{I} \mathrm{m}\left(\hat{J}_{x}^{21, k}-i \hat{J}_{y}^{21, k}\right) \\
& -\frac{1}{2}\left(D_{x}^{+}\right)^{T}\left[\hat{n}_{2}^{k} \circ D_{x}^{+}\left(\hat{A}_{2}^{k+1}-\hat{V}_{s}^{k+1}\right)\right]-\frac{1}{2}\left(D_{x}^{-}\right)^{T}\left[\hat{n}_{2}^{k} \circ D_{x}^{-}\left(\hat{A}_{2}^{k+1}-\hat{V}_{s}^{k+1}\right)\right] \\
& -\frac{1}{2}\left(D_{y}^{+}\right)^{T}\left[\hat{n}_{2}^{k} \circ D_{y}^{+}\left(\hat{A}_{2}^{k+1}-\hat{V}_{s}^{k+1}\right)\right]-\frac{1}{2}\left(D_{y}^{-}\right)^{T}\left[\hat{n}_{2}^{k} \circ D_{y}^{-}\left(\hat{A}_{2}^{k+1}-\hat{V}_{s}^{k+1}\right)\right] \\
& -\alpha \mathcal{R e}\left\{\widetilde{D}_{x}^{T}\left[\hat{n}_{21}^{k} \circ\left(\hat{A}_{1}^{k+1}-\hat{A}_{2}^{k+1}\right)\right]\right\}+\alpha \mathcal{R e}\left\{i \widetilde{D}_{y}^{T}\left[\hat{n}_{21}^{k} \circ\left(\hat{A}_{1}^{k+1}-\hat{A}_{2}^{k+1}\right)\right]\right\} \\
& +\alpha \mathcal{R e}\left\{\hat{n}_{21}^{k} \circ\left[\widetilde{D}_{x}\left(\hat{A}_{1}^{k+1}+\hat{A}_{2}^{k+1}-2 \hat{V}_{s}^{k+1}\right)\right]\right\}
\end{aligned}
$$




$$
\begin{aligned}
& -\alpha \mathcal{R e}\left\{i \hat{n}_{21}^{k} \circ\left[\widetilde{D}_{y}\left(\hat{A}_{1}^{k+1}+\hat{A}_{2}^{k+1}-2 \hat{V}_{s}^{k+1}\right)\right]\right\}=0, \\
& -\gamma^{2} \Delta_{d i r} \hat{V}_{s}^{k+1}=\hat{n}_{1}\left(\hat{A}_{1}^{k+1}, \hat{A}_{2}^{k+1}\right)+\hat{n}_{2}\left(\hat{A}_{1}^{k+1}, \hat{A}_{2}^{k+1}\right), \\
& H\left(\hat{A}_{1}^{k+1}, \hat{A}_{2}^{k+1}\right)\left(\begin{array}{c}
\hat{\psi}_{l}^{1, k+1} \\
\hat{\psi}_{l}^{2, k+1}
\end{array}\right)=\lambda_{l}^{k+1}\left(\begin{array}{c}
\hat{\psi}_{l}^{1, k+1} \\
\hat{\psi}_{l}^{2, k+1}
\end{array}\right), \\
& \hat{n}_{1}\left(\hat{A}_{1}^{k+1}, \hat{A}_{2}^{k+1}\right)=\sum_{l} e^{-\lambda_{l}^{k+1}} \hat{\psi}_{l}^{1, k+1} \circ \overline{\hat{\psi}_{l}^{1, k+1}}, \\
& \hat{n}_{2}\left(\hat{A}_{1}^{k+1}, \hat{A}_{2}^{k+1}\right)=\sum_{l} e^{-\lambda_{l}^{k+1}} \hat{\psi}_{l}^{2, k+1} \circ \overline{\hat{\psi}_{l}^{2, k+1}}, \\
& \hat{n}_{21}^{k}=\sum_{l} e^{-\lambda_{l}^{k}} \hat{\psi}_{l}^{2, k} \circ \overline{\hat{\psi}_{l}^{1, k}} \\
& \hat{J}_{21}^{x, k}=-\frac{i \varepsilon}{2} \sum_{l} e^{-\lambda_{l}^{k}}\left[D_{x}\left(\hat{\psi}_{l}^{2, k}\right) \circ \overline{\hat{\psi}_{l}^{1, k}}-\hat{\psi}_{l}^{2, k} \circ D_{x}\left({\hat{\psi_{l}^{1, k}}}_{l}^{1,}\right),\right.
\end{aligned}
$$

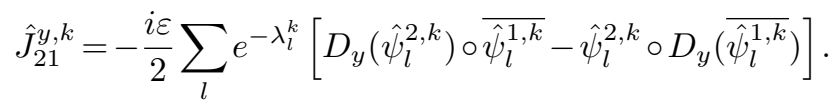

Here, the operator "o" symbolizes the component by component multiplication of two vectors in $\mathbb{C}^{P}$, and the Hamiltonian $H\left(\hat{A}^{k+1}\right)$ is given by

$$
\begin{aligned}
& H\left(\hat{A}_{1}^{k+1}, \hat{A}_{2}^{k+1}\right) \\
= & \left(\begin{array}{cc}
-\frac{\varepsilon^{2}}{2} \Delta_{d i r}+\operatorname{dg}\left(\hat{V}_{e x t, 1}+\hat{A}_{1}^{k+1}\right) & \varepsilon^{2} \alpha\left(D_{x}-i D_{y}\right) \\
-\varepsilon^{2} \alpha\left(D_{x}+i D_{y}\right) & -\frac{\varepsilon^{2}}{2} \Delta_{d i r}+\operatorname{dg}\left(\hat{V}_{e x t, 2}+\hat{A}_{2}^{k+1}\right)
\end{array}\right),
\end{aligned}
$$

where $\operatorname{dg}(\hat{f})$ stands for a diagonal $P \times P$ matrix whose diagonal elements are the components $f_{p}$ of $\hat{f}$. The scheme (4.2)-(4.10) is consistent with the continuous model (2.8)(2.14). It is of first order in time and of second order in space. Due to its rather implicit nature, the scheme (4.2)-(4.10) shows better stability properties than the forward Euler scheme (c.f. numerical tests, Section 5). The solution $\left(\hat{A}_{1}^{k+1}, \hat{A}_{2}^{k+1}, \hat{V}_{s}^{k+1}\right)$ of the system (4.2)-(4.10) is the minimizer of the following discrete functional $\widehat{\mathcal{F}}\left(\hat{A}_{1}, \hat{A}_{2}, \hat{V}_{s}\right): \mathbb{R}^{3 P} \rightarrow \mathbb{R}$ :

$$
\begin{aligned}
\widehat{\mathcal{F}}\left(\hat{A}_{1}, \hat{A}_{2}, \hat{V}_{s}\right):= & \widehat{\mathcal{G}}\left(\hat{A}_{1}, \hat{A}_{2}\right)+\widehat{\mathcal{F}}_{1}\left(\hat{A}_{1}, \hat{A}_{2}, \hat{V}_{s}\right) \\
& +\widehat{\mathcal{F}}_{2}\left(\hat{A}_{1}, \hat{A}_{2}, \hat{V}_{s}\right)+\widehat{\mathcal{F}}_{3}\left(\hat{A}_{1}, \hat{A}_{2}, \hat{V}_{s}\right)+\widehat{\mathcal{F}}_{4}\left(\hat{A}_{1}, \hat{A}_{2}\right),
\end{aligned}
$$

where

$$
\widehat{\mathcal{G}}\left(\hat{A}_{1}, \hat{A}_{2}\right):=\sum_{l=1}^{2 P} e^{-\lambda_{l}\left(\hat{A}_{1}, \hat{A}_{2}\right)}
$$




$$
\begin{aligned}
\widehat{\mathcal{F}}_{1}\left(\hat{A}_{1}, \hat{A}_{2}, \hat{V}_{s}\right):=\frac{\Delta t}{4}\left[\left(\hat{n}_{1}^{k} \circ D_{x}^{+}\left(\hat{A}_{1}-\hat{V}_{s}\right), D_{x}^{+}\left(\hat{A}_{1}-\hat{V}_{s}\right)\right)_{P}\right. \\
+\left(\hat{n}_{1}^{k} \circ D_{x}^{-}\left(\hat{A}_{1}-\hat{V}_{s}\right), D_{x}^{-}\left(\hat{A}_{1}-\hat{V}_{s}\right)\right)_{P}+\left(\hat{n}_{1}^{k} \circ D_{y}^{+}\left(\hat{A}_{1}-\hat{V}_{s}\right), D_{y}^{+}\left(\hat{A}_{1}-\hat{V}_{s}\right)\right)_{P} \\
+\left(\hat{n}_{1}^{k} \circ D_{y}^{-}\left(\hat{A}_{1}-\hat{V}_{s}\right), D_{y}^{-}\left(\hat{A}_{1}-\hat{V}_{s}\right)\right)_{P}+\left(\hat{n}_{2}^{k} \circ D_{x}^{+}\left(\hat{A}_{2}-\hat{V}_{s}\right), D_{x}^{+}\left(\hat{A}_{2}-\hat{V}_{s}\right)\right)_{P} \\
+\left(\hat{n}_{2}^{k} \circ D_{x}^{-}\left(\hat{A}_{2}-\hat{V}_{s}\right), D_{x}^{-}\left(\hat{A}_{2}-\hat{V}_{s}\right)\right)_{P}+\left(\hat{n}_{2}^{k} \circ D_{y}^{+}\left(\hat{A}_{2}-\hat{V}_{s}\right), D_{y}^{+}\left(\hat{A}_{2}-\hat{V}_{s}\right)\right)_{P} \\
\left.+\left(\hat{n}_{2}^{k} \circ D_{y}^{-}\left(\hat{A}_{2}-\hat{V}_{s}\right), D_{y}^{-}\left(\hat{A}_{2}-\hat{V}_{s}\right)\right)_{P}\right], \\
\widehat{\mathcal{F}}_{2}\left(\hat{A}_{1}, \hat{A}_{2}, \hat{V}_{s}\right):=\left(\hat{n}_{1}^{k}, \hat{A}_{1}-\hat{V}_{s}\right)_{P}+\left(\hat{n}_{2}^{k}, \hat{A}_{2}-\hat{V}_{s}\right)_{P} \\
\quad+\frac{\gamma^{2}}{2}\left[\left(D_{x}^{b} \hat{V}_{s}, D_{x}^{b} \hat{V}_{s}\right)_{P}+\left(D_{y}^{b} \hat{V}_{s}, D_{y}^{b} \hat{V}_{s}\right)_{P}\right] \\
\quad+\frac{\Delta y}{\Delta x} \sum_{i=1}^{N} V_{s, i M}^{2}+\frac{\Delta x}{\Delta y} \sum_{j=1}^{M} V_{s, N j}^{2} \\
\widehat{\mathcal{F}}_{3}\left(\hat{A}_{1}, \hat{A}_{2}, \hat{V}_{s}\right):=\alpha \Delta t \mathcal{R e}\left[\left(\hat{n}_{21}^{k} \circ\left(\hat{A}_{1}-\hat{A}_{2}\right), \widetilde{D}_{x}\left(\hat{A}_{1}+\hat{A}_{2}-2 \hat{V}_{s}\right)\right)_{P}\right. \\
\left.-i\left(\hat{n}_{21}^{k} \circ\left(\hat{A}_{1}-\hat{A}_{2}\right), \widetilde{D}_{y}\left(\hat{A}_{1}+\hat{A}_{2}-2 \hat{V}_{s}\right)\right)_{P}\right] \\
\widehat{\mathcal{F}}_{4}\left(\hat{A}_{1}, \hat{A}_{2}\right):=\frac{\alpha \Delta t}{\varepsilon} \mathcal{I m}\left[\left(\left(\hat{A}_{1}-\hat{A}_{2}\right) \circ\left(\hat{A}_{1}-\hat{A}_{2}\right), \hat{J}_{x}^{21, k}-i \hat{J}_{y}^{21, k}\right)_{P}\right]
\end{aligned}
$$

and the further discretization matrices $D_{x}^{b}$ and $D_{y}^{b}$ are also defined in Appendix D. Using the relation

$$
\begin{aligned}
-\left(\hat{V}_{s}, \Delta_{d i r} \hat{V}_{s}\right)_{P}= & \left(D_{x}^{b} \hat{V}_{s}, D_{x}^{b} \hat{V}_{s}\right)_{P}+\left(D_{y}^{b} \hat{V}_{s}, D_{y}^{b} \hat{V}_{s}\right)_{P} \\
& +\frac{\Delta y}{\Delta x} \sum_{i=1}^{N} V_{s, i M}^{2}+\frac{\Delta x}{\Delta y} \sum_{j=1}^{M} V_{s, N j}^{2}
\end{aligned}
$$

it can be readily verified that a solution $\left(\hat{A}_{1}^{k+1}, \hat{A}_{2}^{k+1}, \hat{V}_{s}^{k+1}\right)$ of (4.2)-(4.10) satisfies

$$
\mathrm{d} \widehat{\mathcal{F}}\left(\hat{A}_{1}^{k+1}, \hat{A}_{2}^{k+1}, \hat{V}_{s}^{k+1}\right)\left(\delta \hat{A}, \delta \hat{V}_{s}\right)=0 \quad \forall\left(\delta \hat{A}_{1}, \delta \hat{A}_{2}, \delta \hat{V}_{s}\right) \in \mathbb{R}^{3 P} .
$$

4.2. A second fully discrete system (scheme 2). We chose the following space discretization of the forward Euler scheme (3.13)-(3.18):

$$
\begin{aligned}
& \frac{\hat{n}_{1}^{k+1}-\hat{n}_{1}^{k}}{\Delta t}-\frac{2 \alpha}{\varepsilon}\left(\hat{A}_{1}^{k}-\hat{A}_{2}^{k}\right) \circ \mathcal{I} \operatorname{m}\left(\hat{J}_{x}^{21, k}-i \hat{J}_{y}^{21, k}\right) \\
& -\frac{1}{2}\left(D_{x}^{+}\right)^{T}\left[\hat{n}_{1}^{k} \circ D_{x}^{+}\left(\hat{A}_{1}^{k}-\hat{V}_{s}^{k}\right)\right]-\frac{1}{2}\left(D_{x}^{-}\right)^{T}\left[\hat{n}_{1}^{k} \circ D_{x}^{-}\left(\hat{A}_{1}^{k}-\hat{V}_{s}^{k}\right)\right] \\
& -\frac{1}{2}\left(D_{y}^{+}\right)^{T}\left[\hat{n}_{1}^{k} \circ D_{y}^{+}\left(\hat{A}_{1}^{k}-\hat{V}_{s}^{k}\right)\right]-\frac{1}{2}\left(D_{y}^{-}\right)^{T}\left[\hat{n}_{1}^{k} \circ D_{y}^{-}\left(\hat{A}_{1}^{k}-\hat{V}_{s}^{k}\right)\right] \\
& -\alpha \mathcal{R e}\left\{\widetilde{D}_{x}^{T}\left[\hat{n}_{21}^{k} \circ\left(\hat{A}_{1}^{k}-\hat{A}_{2}^{k}\right)\right]\right\}+\alpha \mathcal{R e}\left\{i \widetilde{D}_{y}^{T}\left[\hat{n}_{21}^{k} \circ\left(\hat{A}_{1}^{k}-\hat{A}_{2}^{k}\right)\right]\right\} \\
& -\alpha \mathcal{R e}\left\{\hat{n}_{21}^{k} \circ\left[\widetilde{D}_{x}\left(\hat{A}_{1}^{k}+\hat{A}_{2}^{k}-2 \hat{V}_{s}^{k}\right)\right]\right\}+\alpha \mathcal{R e}\left\{i \hat{n}_{21}^{k} \circ\left[\widetilde{D}_{y}\left(\hat{A}_{1}^{k}+\hat{A}_{2}^{k}-2 \hat{V}_{s}^{k}\right)\right]\right\}
\end{aligned}
$$


$=0$,

$$
\begin{aligned}
& \frac{\hat{n}_{2}^{k+1}-\hat{n}_{2}^{k}}{\Delta t}+\frac{2 \alpha}{\varepsilon}\left(\hat{A}_{1}^{k}-\hat{A}_{2}^{k}\right) \circ \mathcal{I} \mathrm{m}\left(\hat{J}_{x}^{21, k}-i \hat{J}_{y}^{21, k}\right) \\
& -\frac{1}{2}\left(D_{x}^{+}\right)^{T}\left[\hat{n}_{2}^{k} \circ D_{x}^{+}\left(\hat{A}_{2}^{k}-\hat{V}_{s}^{k}\right)\right]-\frac{1}{2}\left(D_{x}^{-}\right)^{T}\left[\hat{n}_{2}^{k} \circ D_{x}^{-}\left(\hat{A}_{2}^{k}-\hat{V}_{s}^{k}\right)\right] \\
& -\frac{1}{2}\left(D_{y}^{+}\right)^{T}\left[\hat{n}_{2}^{k} \circ D_{y}^{+}\left(\hat{A}_{2}^{k}-\hat{V}_{s}^{k}\right)\right]-\frac{1}{2}\left(D_{y}^{-}\right)^{T}\left[\hat{n}_{2}^{k} \circ D_{y}^{-}\left(\hat{A}_{2}^{k}-\hat{V}_{s}^{k}\right)\right] \\
& -\alpha \mathcal{R e}\left\{\widetilde{D}_{x}^{T}\left[\hat{n}_{21}^{k} \circ\left(\hat{A}_{1}^{k}-\hat{A}_{2}^{k}\right)\right]\right\}+\alpha \mathcal{R e}\left\{i \widetilde{D}_{y}^{T}\left[\hat{n}_{21}^{k} \circ\left(\hat{A}_{1}^{k}-\hat{A}_{2}^{k}\right)\right]\right\} \\
& +\alpha \mathcal{R e}\left\{\hat{n}_{21}^{k} \circ\left[\widetilde{D}_{x}\left(\hat{A}_{1}^{k}+\hat{A}_{2}^{k}-2 \hat{V}_{s}^{k}\right)\right]\right\}-\alpha \mathcal{R} \mathrm{e}\left\{i \hat{n}_{21}^{k} \circ\left[\widetilde{D}_{y}\left(\hat{A}_{1}^{k}+\hat{A}_{2}^{k}-2 \hat{V}_{s}^{k}\right)\right]\right\} \\
& =0 \text {, } \\
& -\gamma^{2} \Delta_{d i r} \hat{V}_{s}^{k}=\hat{n}_{1}^{k}+\hat{n}_{2}^{k} \\
& H\left(\hat{A}_{1}^{k}, \hat{A}_{2}^{k}\right)\left(\begin{array}{c}
\hat{\psi}_{l}^{1, k} \\
\hat{\psi}_{l}^{2, k}
\end{array}\right)=\lambda_{l}^{k}\left(\begin{array}{c}
\hat{\psi}_{l}^{1, k} \\
\hat{\psi}_{l}^{2, k}
\end{array}\right), \\
& \hat{n}_{1}^{k}=\sum_{l} e^{-\lambda_{l}^{k}} \hat{\psi}_{l}^{1, k} \circ \overline{\hat{\psi}_{l}^{1, k}}, \quad \hat{n}_{2}^{k}=\sum_{l} e^{-\lambda_{l}^{k}} \hat{\psi}_{l}^{2, k} \circ \overline{\hat{\psi}_{l}^{2, k}}, \\
& \hat{n}_{21}^{k}=\sum_{l} e^{-\lambda_{l}^{k}} \hat{\psi}_{l}^{2, k} \circ \overline{\hat{\psi}_{l}^{1, k}}
\end{aligned}
$$

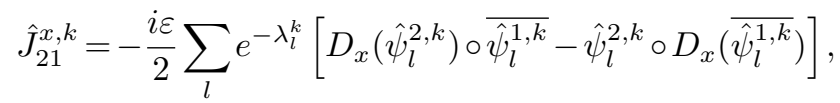

$$
\begin{aligned}
& \hat{J}_{21}^{y, k}=-\frac{i \varepsilon}{2} \sum_{l} e^{-\lambda_{l}^{k}}\left[D_{y}\left(\hat{\psi}_{l}^{2, k}\right) \circ{\hat{\psi_{l}^{1, k}}}_{l}-\hat{\psi}_{l}^{2, k} \circ D_{y}\left(\overline{\hat{\psi}_{l}^{1, k}}\right)\right] \text {. }
\end{aligned}
$$

Here, the Hamiltonian $H$ is the same discrete Hamiltonian (4.11) as in the first fully discrete scheme. Clearly, the scheme (4.19)-(4.26) is consistent with the continuous model (2.8)-(2.14). It is of first order in time and of second order in space. A drawback of the explicit nature of the forward Euler scheme (3.13)-(3.18) is that its full discretization is only conditionally stable; i.e. the space-time grid must be chosen in such a way that a CFL condition is fulfilled.

The solution of this scheme requires the inversion of the non-local relation (4.22)(4.23) at each time step. For this, let us define the discrete version $\widehat{\mathcal{G}}_{n}: \mathbb{R}^{2 P} \rightarrow \mathbb{R}$ of $(3.19)$,

$$
\widehat{\mathcal{G}}_{n}\left(\hat{A}_{1}, \hat{A}_{2}\right):=\widehat{\mathcal{G}}\left(\hat{A}_{1}, \hat{A}_{2}\right)+\left(\hat{n}_{1}^{k}, \hat{A}_{1}\right)_{P}+\left(\hat{n}_{2}^{k}, \hat{A}_{2}\right)_{P} .
$$

It can be easily verified that the first derivative of this functional is given by

$$
\begin{aligned}
\mathrm{d} \widehat{\mathcal{G}}_{n}\left(\hat{A}_{1}, \hat{A}_{2}\right)\left(\delta \hat{A}_{1}, \delta \hat{A}_{2}\right)= & \left(-\sum_{l} e^{-\lambda_{l}^{k}} \hat{\psi}_{l}^{1, k} \circ \overline{\hat{\psi}_{l}^{1, k}}+\hat{n}_{1}^{k}, \delta \hat{A}_{1}\right)_{P} \\
& +\left(-\sum_{l} e^{-\lambda_{l}^{k}} \hat{\psi}_{l}^{2, k} \circ \overline{\hat{\psi}_{l}^{2, k}}+\hat{n}_{2}^{k}, \delta \hat{A}_{2}\right)_{P},
\end{aligned}
$$


whose zeros are hence the solutions of (4.22)-(4.23).

REMARK 4.1. The equations (3.13)-(3.14) contain a term of conservative form $\nabla \cdot\left(n_{j} \nabla A_{j}\right)$. Therefore, appropriate discretizations for conservation laws [11], such as Lax-Friedrichs, should be used to ensure numerical stability. Nevertheless, we employed a forward Euler scheme with central difference in space which is known to be unconditionally unstable for (linear) hyperbolic equations. In the numerics Section 5, the explicit scheme is found to be stable for small values of the semiclassical parameter, i.e. $\varepsilon \leq 0.5$. An explanation for the observed stability can be given by regarding the Lagrange multipliers $A_{j}^{\varepsilon}=A_{j}^{\varepsilon}\left(n_{1}, n_{2}\right)$ in the semi-classical limit $\varepsilon \rightarrow 0$. As described in [2], the correct semi classical expansion reads

$$
A_{j}^{\varepsilon}\left(n_{1}, n_{2}\right)=-\log n_{j}+\frac{\varepsilon^{2}}{6} \frac{\Delta \sqrt{n_{j}}}{\sqrt{n_{j}}}+\mathcal{O}\left(\varepsilon^{2} \alpha^{2}\right) .
$$

Therefore, in the limit $\varepsilon \rightarrow 0$, the conservative term reads

$$
\nabla \cdot\left(n_{j}^{\varepsilon} \nabla A_{j}^{\varepsilon}\right) \quad \stackrel{\varepsilon \rightarrow 0}{\longrightarrow} \quad-\nabla \cdot\left(n_{j} \nabla \log n_{j}\right) .
$$

Hence, for small $\varepsilon$, equations (3.13)-(3.14) resemble a heat equation or a drift-diffusion equation, respectively, where the diffusive term is written in the non-standard form (4.30). In this case, a forward Euler scheme with central finite difference space derivatives is stable with respect to a CFL condition of the form $\Delta t \leq d \Delta x^{2}$ for some constant $d$.

4.3. Initialization of scheme 1. As was briefly mentioned in Section 2, a natural way to initialize the system (4.2)-(4.10) would be to start from given initial chemical potentials $\hat{A}_{1}^{0}$ and $\hat{A}_{2}^{0}$, compute the corresponding spin and current densities, and subsequently begin the iteration. However, from an experimental point of view it is more appealing to start from the initial spin densities $\hat{n}_{1}^{0}$ and $\hat{n}_{2}^{0}$. The problem in the latter approach is the lack of information about the initial spin-mixing quantities $\hat{n}_{21}^{0}$, $\hat{J}_{21}^{x, 0}$, and $\hat{J}_{21}^{y, 0}$ which are not directly related to the spin densities. At $t=t^{0}$ it is thus necessary to do a half step of scheme 2, which means minimizing the functional (4.27) in order to obtain the chemical potentials corresponding to the initial spin densities $\hat{n}_{1}^{0}$ and $\hat{n}_{2}^{0}$. One can then proceed according to scheme 1 .

\section{Numerical results}

This part deals with the numerical study of the two fully discrete schemes introduced in the previous section. In the spin-less case, it is well known that the steady-state solutions $\left(\partial_{t} n=0\right)$ of quantum drift-diffusion models are solutions of the corresponding stationary Schrödinger-Poisson problem $[15,16]$. We shall check whether this convergence in the long-time limit is achieved by the numerical schemes developed in this work for the spin-dependent case. Tests are performed for different values of the semiclassical parameters $\varepsilon$, the Rashba coupling parameter $\alpha$ and the discretization parameters $\Delta x$ and $\Delta t$. Moreover, the performance of the semi-implicit scheme 1 (c.f. Section 4.1) will be compared to the performance of the explicit scheme 2 (c.f. Section 4.2). In what follows, the spin-dependent external potentials are chosen to be

$$
V_{\text {ext }, 1}=0.0, \quad V_{\text {ext }, 2}=\sin (2 \pi x) \sin (2 \pi y) .
$$

Using these external potentials, steady-state solutions were obtained by solving the stationary Schrödinger-Poisson (SP) system (4.4)-(4.7). In the discrete Hamiltonian 
(4.11), we set $\hat{A}_{1}^{k+1}=\hat{A}_{2}^{k+1}=\hat{V}_{s}^{k}$ where $k$ now denotes the index of the SP-iteration. We use a space grid with $N=M=41$ points in each direction and set $\gamma=1$ (throughout this section). During SP-iteration, the total mass density was renormalized to 1.0 after each solution of the eigenvalue problem. We set $\hat{V}_{s}^{0}=0.0$ and iterated until the change in the spin densities was less than $10^{-6}$ from one iteration to the next. Obtained solutions are denoted with an index 'SP' and are depicted in Figure 5.1 (for $\alpha=1$ and different values $\varepsilon$ ) and in Figure 5.2 (for $\varepsilon=0.2$ and different values $\alpha$ ).

In view of the the external potentials given in (5.1), it is reasonable to obtain maxima of the total mass density $n_{t o t}^{S P}=n_{1}^{S P}+n_{2}^{S P}$ at the minima of $V_{\text {ext, }}$, namely $(x, y)=(1 / 4,3 / 4)$ and $(x, y)=(3 / 4,1 / 4)$. Clearly, the spin polarization $n_{\text {pol }}^{S P}=n_{1}^{S P}-n_{2}^{S P}$ has minima at those points. In Figure 5.1, we remark that the larger the semiclassical parameter $\varepsilon$ becomes the less apparent is the influence of the external potentials on the steady state. This is expected since, in the quantum regime, electrons have the ability to 'tunnel' potential barriers which therefore tend to have a lesser impact on the electron distribution. Additionally, from Figure 5.2, we learn that increased values of the Rashba coupling parameter $\alpha$ lead to lesser spin polarization in the steady state. This is physically reasonable because Rashba spin-orbit coupling is a source of spin depolarization [21]. On the other hand, the total mass density is hardly affected by a change of $\alpha$.

Let us present a few computational details concerning the numerics. The developed algorithms were implemented in the FORTRAN 90 language. Eigenvalue problems were solved using the routine 'zheev.f90' from the LAPACK library. The solution of scheme 1, equations (4.2)-(4.10), was achieved by minimizing the discrete functional (4.12) at each time step $t_{k}, k>0$. At $t_{0}$, the system was initialized as detailed in Subsection 4.3. Each minimization problem was solved by a conjugate gradient method in the parameter space $\mathbb{R}^{3 P}$ (or $\mathbb{R}^{2 P}$ for scheme 2 , respectively). We denote vectors in the parameter space by capital letters, i.e. $X=\left(\hat{A}_{1}, \hat{A}_{2}, \hat{V}_{s}\right), X \in \mathbb{R}^{3 P}$ in scheme 1 and $X=\left(\hat{A}_{1}, \hat{A}_{2}\right)$, $X \in \mathbb{R}^{2 P}$ in scheme 2 . In the parameter space, the gradient is denoted by ' $\nabla_{X}$ ' and the usual euclidean scalar product is denoted by ' $'$ '. In order to find the line minimum of $\nabla_{X} \widehat{\mathcal{F}} \cdot Y_{n}$, where $Y_{n}$ denotes the search direction $\left(\left|Y_{n}\right|=1\right)$ during the $n$-th step of the conjugate gradient scheme, a Newton method was employed. The derivative of $\nabla_{X} \widehat{\mathcal{F}} \cdot Y_{n}$ in the direction $Y_{n}$ was computed numerically with a forward discretization and the small step size $\varepsilon_{N T}=10^{-3}$,

$$
\left(\nabla_{X} \widehat{\mathcal{F}}(X) \cdot Y_{n}\right)^{\prime} \approx \frac{\nabla_{X} \widehat{\mathcal{F}}\left(X+\varepsilon_{N T} Y_{n}\right) \cdot Y_{n}-\nabla_{X} \widehat{\mathcal{F}}(X) \cdot Y_{n}}{\varepsilon_{N T}} .
$$

The same method was applied to the functional $\widehat{\mathcal{G}}_{n}$ in scheme 2 . The Newton method considered converged when $\left|\nabla_{X} \widehat{\mathcal{F}}(X) \cdot Y_{n}\right|<10^{-10}$. We established two convergence criteria for the conjugate gradient method. In scheme 1, the functional (4.12) was considered optimized when the maximal change in the vector $\left(\hat{A}_{1}, \hat{A}_{2}, \hat{V}_{s}\right)$ was less than $10^{-5}$ from one conjugate gradient step to the next. On the other hand, in scheme 2 and during initialization, the functional (4.27) was considered optimized when

$$
\left\|\nabla_{X} \widehat{\mathcal{G}}_{n}\right\|_{\infty}<10^{-5}
$$

In all tests performed, the initial spin densities were two Gaussians centered at $(x, y)=$ (0.5,0.5),

$$
n_{1}^{0}(x, y)=n_{2}^{0}(x, y)=\frac{1}{0.12 \pi} \exp \left(-\frac{(x-0.5)^{2}}{0.06}-\frac{(y-0.5)^{2}}{0.06}\right)
$$



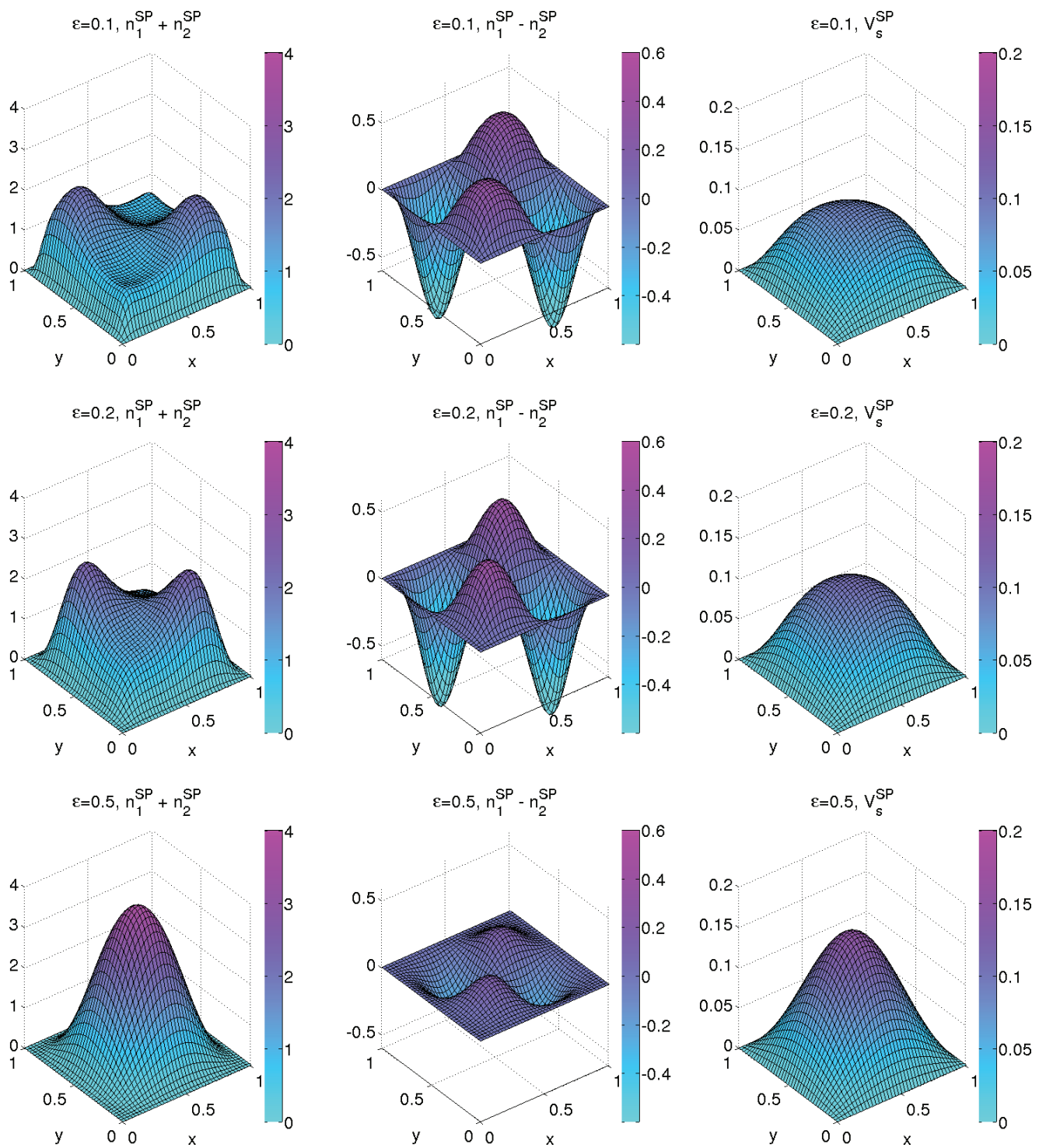

$$
\varepsilon=1.0, n_{1}^{S P}+n_{2}^{S P}
$$
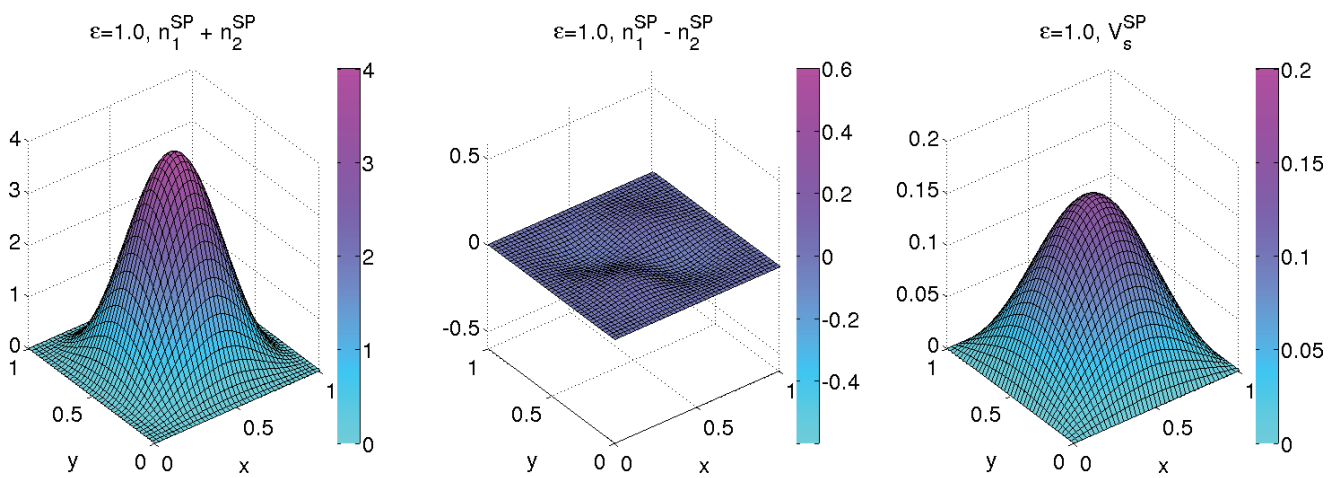

FIG. 5.1. Solutions of the stationary Schrödinger-Poisson system (4.4)-(4.7) with external potentials (5.1) and $A_{1}=A_{2}=V_{s}$. The number of mesh points is $N=M=41$. Parameters are $\alpha=1.0$ and $\varepsilon$ ranging from 0.1 (line 1) to 1.0 (line 4). Additionally, $n_{1}^{S P}+n_{2}^{S P}$ is the total mass density, $n_{1}^{S P}-n_{2}^{S P}$ denotes the spin polarization, and $V_{s}^{S P}$ is the self-consistent electric potential. 
$\alpha=1.0, n_{1}^{\mathrm{SP}}+\mathrm{n}_{2}^{\mathrm{SP}}$

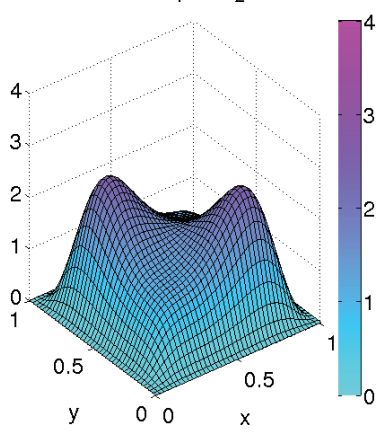

$\alpha=5.0, n_{1}^{\mathrm{SP}}+\mathrm{n}_{2}^{\mathrm{SP}}$

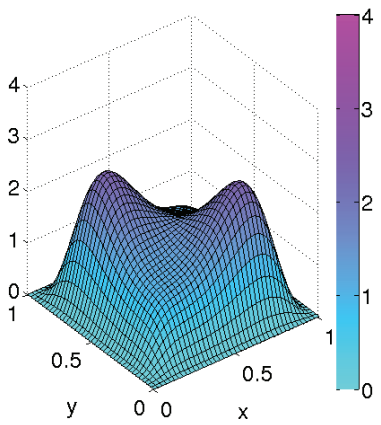

$\alpha=10.0, n_{1}^{\mathrm{SP}}+\mathrm{n}_{2}^{\mathrm{SP}}$

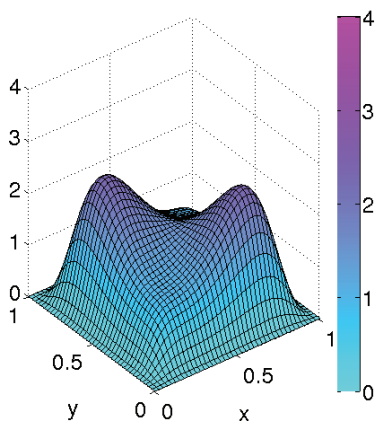

$\alpha=20.0, n_{1}^{\mathrm{SP}}+\mathrm{n}_{2}^{\mathrm{SP}}$

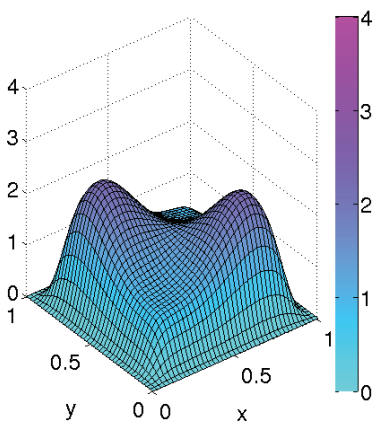

$\alpha=1.0, n_{1}^{\mathrm{SP}}-\mathrm{n}_{2}^{\mathrm{SP}}$

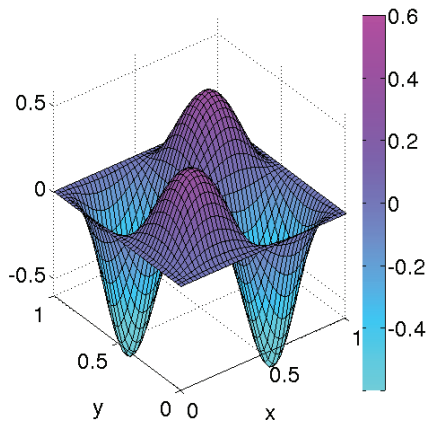

$\alpha=5.0, n_{1}^{\mathrm{SP}}-\mathrm{n}_{2}^{\mathrm{SP}}$

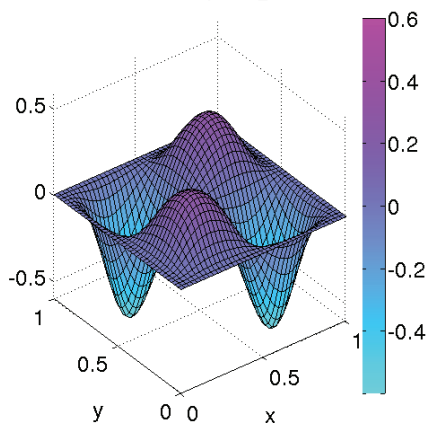

$\alpha=10.0, n_{1}^{S P}-n_{2}^{S P}$

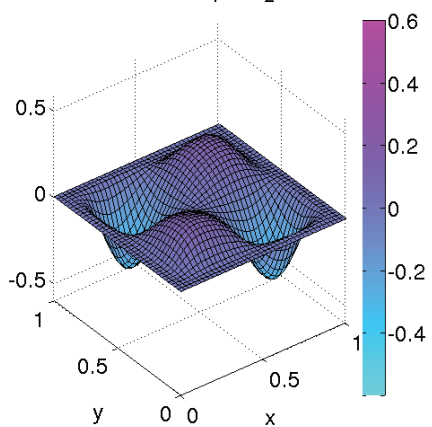

$\alpha=20.0, n_{1}^{S P}-n_{2}^{S P}$

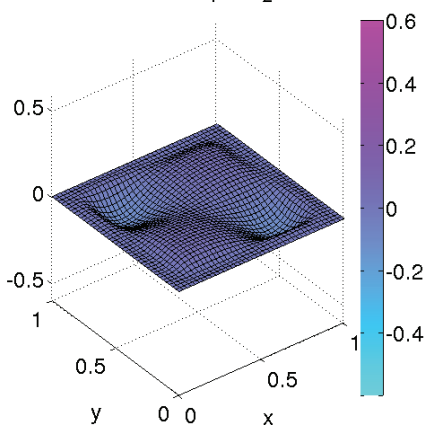

$\alpha=1.0, V_{s}^{S P}$

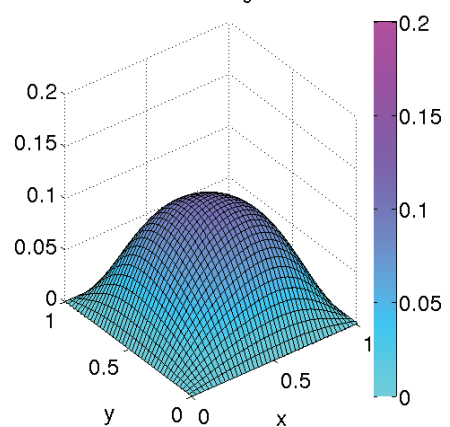

$\alpha=5.0, \mathrm{~V}_{\mathrm{s}}^{\mathrm{SP}}$

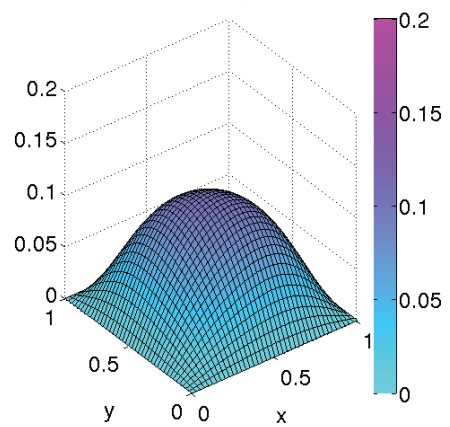

$\alpha=10.0, V_{s}^{S P}$

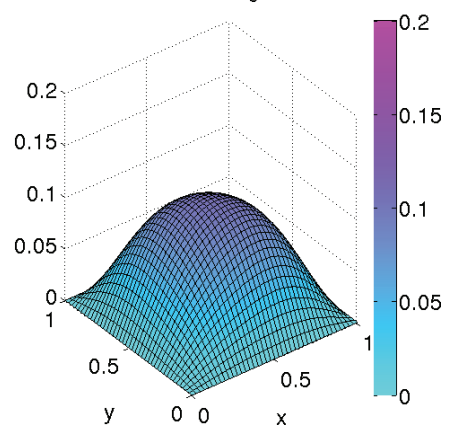

$\alpha=20.0, V_{s}^{S P}$

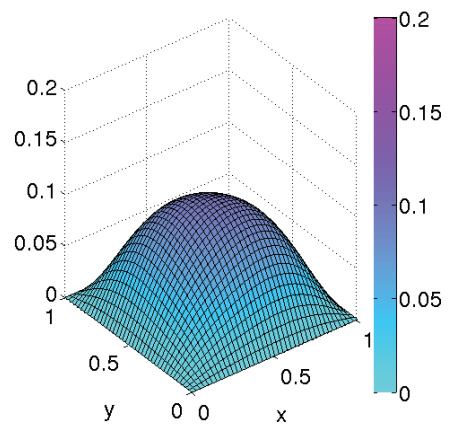

FIG. 5.2. Solutions of the stationary Schrödinger-Poisson system (4.4)-(4.7) with external potentials (5.1) and $A_{1}=A_{2}=V_{s}$. The number of mesh points is $N=M=41$. Parameters are $\varepsilon=0.2$ and $\alpha$ ranging from 1.0 (line 1) to 20.0 (line 4). Additionally, $n_{1}^{S P}+n_{2}^{S P}$ is the total mass density, $n_{1}^{S P}-n_{2}^{S P}$ denotes the spin polarization, and $V_{s}^{S P}$ is the self-consistent electric potential. 
The initial data for $n_{1}$ and $n_{2}$ were discretized according to the conventions at the beginning of Section 4. The initial total mass of the system was 1.0. We recall that, in all simulations, the externally applied potentials are given by (5.1).
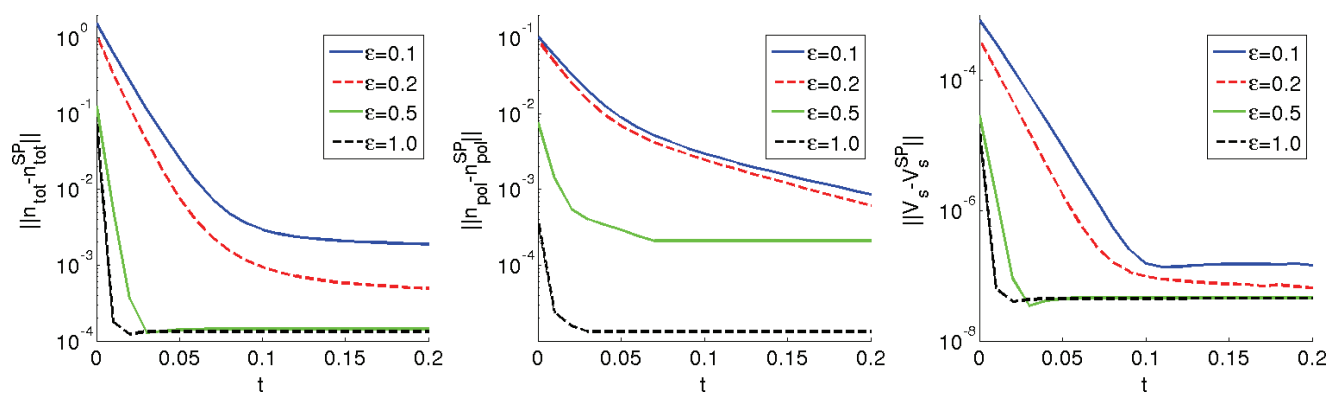

(a) Numerical convergence (in $L^{2}$-norm) for $\alpha=1.0$ (scheme 1 ).
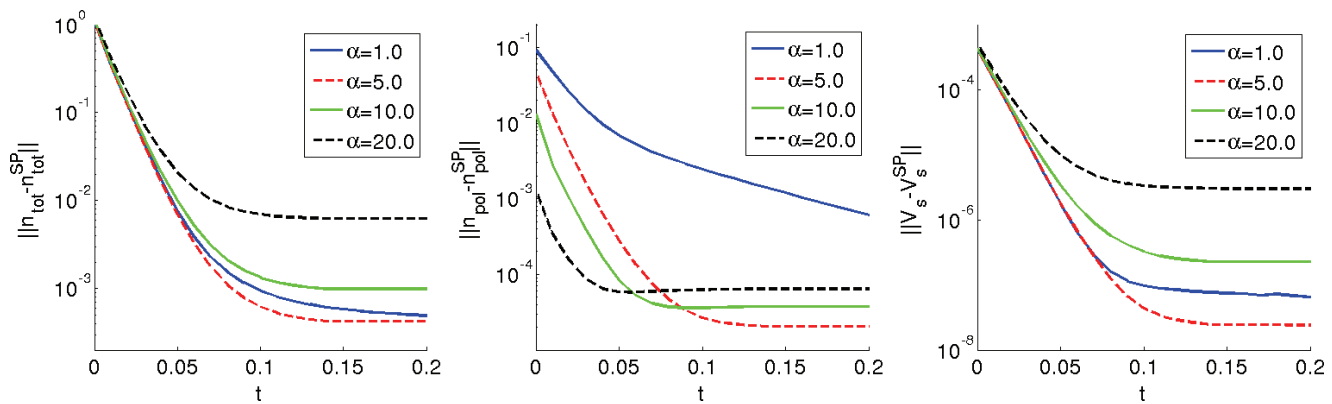

(b) Numerical convergence (in $L^{2}$-norm) for $\varepsilon=0.2$ (scheme 1 ).

FIG. 5.3. Numerical convergence (in $L^{2}$-norm) over time of the initial state (5.4) to the steady states 'SP' (solutions of the stationary Schrödinger-Poisson problem depicted in Figures 5.1 and 5.2) computed with the semi-implicit scheme 1. Simulation results are shown in (a) for different values of $\varepsilon(\alpha=1.0)$ and in (b) for different values of $\alpha(\varepsilon=0.2)$. Numerical parameters were $\Delta x=\Delta y=0.05$ and $\Delta t=10^{-2}$.

5.1. Long-time convergence towards steady state. We shall test the relaxation of the initial data (5.4) towards the steady-state solutions 'SP' shown in Figure 5.1 and Figure 5.2, respectively, for different values of the parameters $\varepsilon$ and $\alpha$. Simulations are performed with the semi-implicit scheme 1, equations (4.2)-(4.10). We choose a time step $\Delta t=10^{-2}$ and simulate until the final time $t_{f}=0.2$, i.e. 20 time steps. The number of grid points in each direction is $N=M=21$, thus $\Delta x=\Delta y=0.05$. The explicit scheme 2 , along with different choices for the time and the space discretization, will be tested in the next subsection.

Figure 5.3 demonstrates the long-time convergence of the Gaussian initial state towards the stationary Schrödinger-Poisson states for various choices of the semiclassical parameter $\varepsilon$ and the Rashba coupling parameter $\alpha$. Convergence is with respect to the $L^{2}$-norm. The three columns of sub-figures show the $L^{2}$-errors over time of the total mass density $\left\|n_{t o t}-n_{t o t}^{S P}\right\|$, of the spin polarization $\left\|n_{p o l}-n_{\text {pol }}^{S P}\right\|$, and of the selfconsistent potential $\left\|V_{s}-V_{s}^{S P}\right\|$, respectively. As can be seen in subfigure (a), relaxation is faster and the reached accuracy (the 'plateau') is more favorable for larger values of $\varepsilon$ (quantum regime). By contrast, from subfigure (b) one obtains that for $\varepsilon=0.2$ the rate of convergence is hardly dependent on the Rashba coupling parameter $\alpha$. Moreover, the 
reached accuracy (plateau) gets worse as $\alpha$ increases.

In order to highlight the obtained long-time convergence, we show the evolution of the total mass density $n_{t o t}=n_{1}+n_{2}$, the spin polarization $n_{p o l}=n_{1}-n_{2}$, and the self-consistent potential $V_{s}$ for parameters $\varepsilon=0.1$ and $\alpha=1.0$ in figures 5.4 and 5.5, respectively.

5.2. Parameter studies for the schemes 1 and 2 . It is the aim of this subsection to compare the performances of the semi-implicit scheme 1, equations (4.2)-(4.10), and the explicit scheme 2, equations (4.19)-(4.26), by means of long-time simulations until the final time $t_{f}=0.2$. Various values of the parameters $\varepsilon$ and $\alpha$ will be tested. Moreover, different space discretizations shall be applied in order to check for numerical convergence as $\Delta x \rightarrow 0^{1}$. As in the previous subsection, the external potentials are given by (5.1) and we use the initial spin densities (5.4).

\begin{tabular}{c|c|c|c|c|c|r} 
scheme & $\varepsilon$ & $\Delta x$ & $\Delta t$ & \#CG (init.) & avg.\#CG & $t_{C P U}$ \\
\hline \hline semi-implicit & 0.1 & 0.10 & $10^{-2}$ & 81 & 79.9 & 1.7 \\
semi-implicit & 0.2 & 0.10 & $10^{-2}$ & 71 & 95.8 & 2.0 \\
semi-implicit & 0.5 & 0.10 & $10^{-2}$ & 159 & 71.1 & 1.3 \\
semi-implicit & 1.0 & 0.10 & $10^{-2}$ & 226 & 45.7 & 1.0 \\
\hline explicit & 0.1 & 0.10 & $10^{-4}$ & 81 & 4.4 & 9.2 \\
explicit & 0.2 & 0.10 & $10^{-4}$ & 71 & 5.1 & 10.3 \\
explicit & 0.5 & 0.10 & $10^{-5}$ & 159 & 1.9 & 31.9 \\
explicit & 1.0 & 0.10 & $10^{-5}$ & 226 & 1.5 & 25.6 \\
\hline \hline semi-implicit & 0.1 & 0.07 & $10^{-2}$ & 118 & 127.6 & 20.0 \\
semi-implicit & 0.2 & 0.07 & $10^{-2}$ & 177 & 168.7 & 23.5 \\
semi-implicit & 0.5 & 0.07 & $10^{-2}$ & 357 & 138.5 & 17.8 \\
semi-implicit & 1.0 & 0.07 & $10^{-2}$ & 410 & 89.9 & 13.4 \\
\hline explicit & 0.1 & 0.07 & $10^{-4}$ & 118 & 5.3 & 78.6 \\
explicit & 0.2 & 0.07 & $5 \cdot 10^{-5}$ & 177 & 6.0 & 154.6 \\
explicit & 0.5 & 0.07 & $10^{-5}$ & 357 & 2.2 & 264.8 \\
explicit & 1.0 & 0.07 & $10^{-6}$ & 410 & 1.2 & 1292.3 \\
\hline \hline semi-implicit & 0.1 & 0.05 & $10^{-2}$ & 195 & 234.1 & 288.7 \\
semi-implicit & 0.2 & 0.05 & $10^{-2}$ & 487 & 326.0 & 358.9 \\
semi-implicit & 0.5 & 0.05 & $10^{-2}$ & 675 & 278.8 & 285.9 \\
semi-implicit & 1.0 & 0.05 & $10^{-2}$ & 1045 & 175.2 & 229.8 \\
\hline \hline
\end{tabular}

TABLE 5.1. Comparison of the performance of the semi-implicit scheme 1, equations (4.2)-(4.10), with the explicit scheme 2, equations (4.19)-(4.26). The computational time $t_{C P U}$ has been normalized to a run with the parameter set in line four of this table. Final simulation time was $t_{f}=0.2$. Moreover, $\alpha=1.0$ and $\Delta x=\Delta y$ in all simulations. Additionally, '\#CG (init.)' stands for the number of conjugate gradient steps during the initialization and 'avg.\#CG' denotes the average number of conjugate gradient steps in one time step. Parameters shown here were used to obtain the results depicted in Figure 5.6.

For $\alpha=1.0$ and various choices of the parameters $\varepsilon$ and $\Delta x$, a comparison regarding the computational cost of the schemes 1 and 2 is given in Table 5.1. The CPU-time $t_{C P U}$

\footnotetext{
${ }^{1}$ We remark that, due to the large number of eigenvalue problems to be solved at each time step, mesh refinement beyond $\Delta x=0.05$ was not feasible. For example, for $N=M=41$, the solution of one eigenvalue problem takes about 1500 seconds which is required approximately 500-1000 times to arrive at $t_{f}=0.2$.
} 

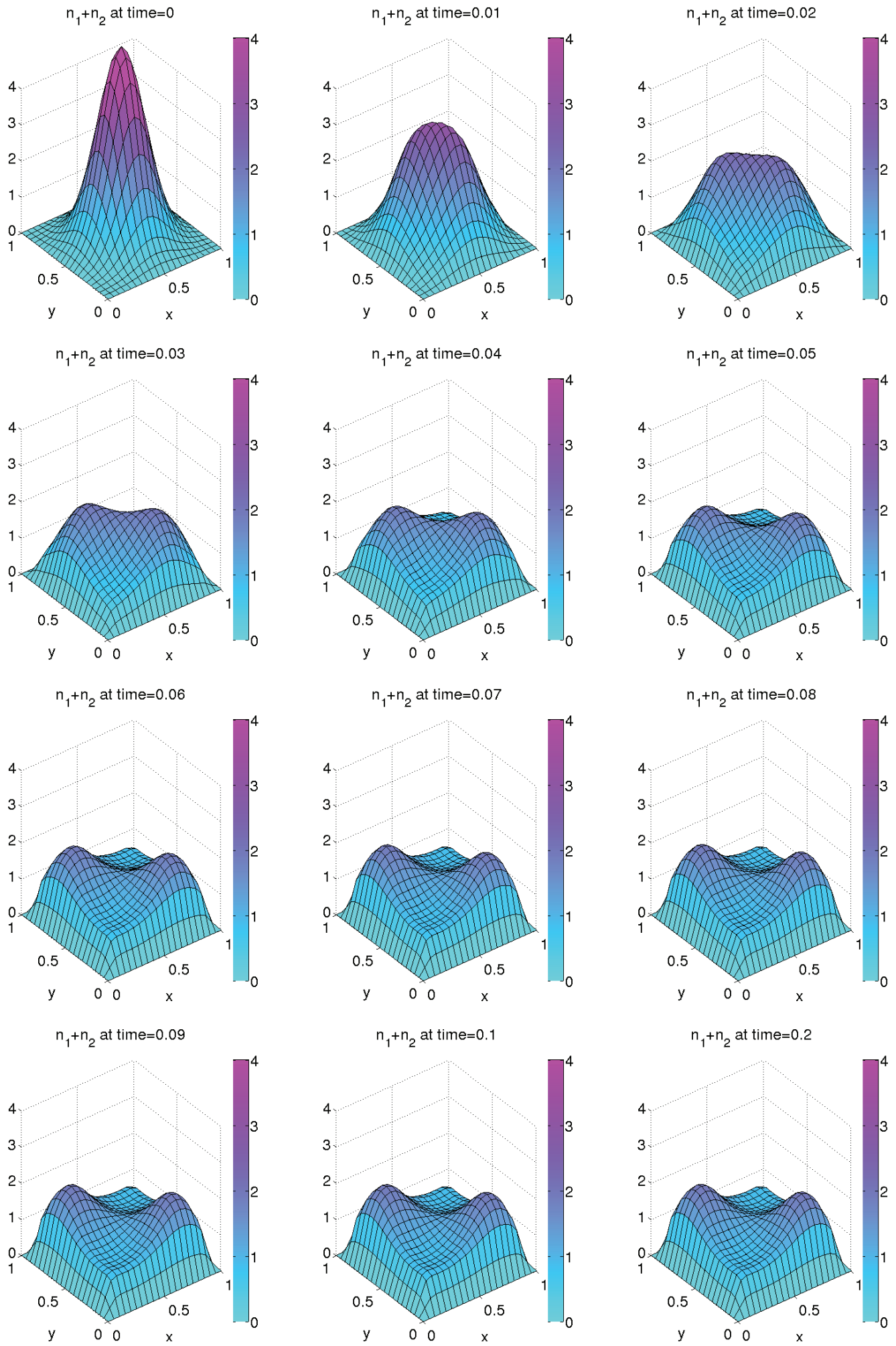

FIG. 5.4. Evolution of the total mass density of the initial state (5.4) towards the steady state 'SP' computed from the stationary Schrödinger-Poisson system depicted in Figure 5.1. Parameters were $\varepsilon=0.1, \alpha=1.0, \Delta x=\Delta y=0.05$, and $\Delta t=10^{-2}$. The simulation was performed with the semi-implicit scheme 1 . 

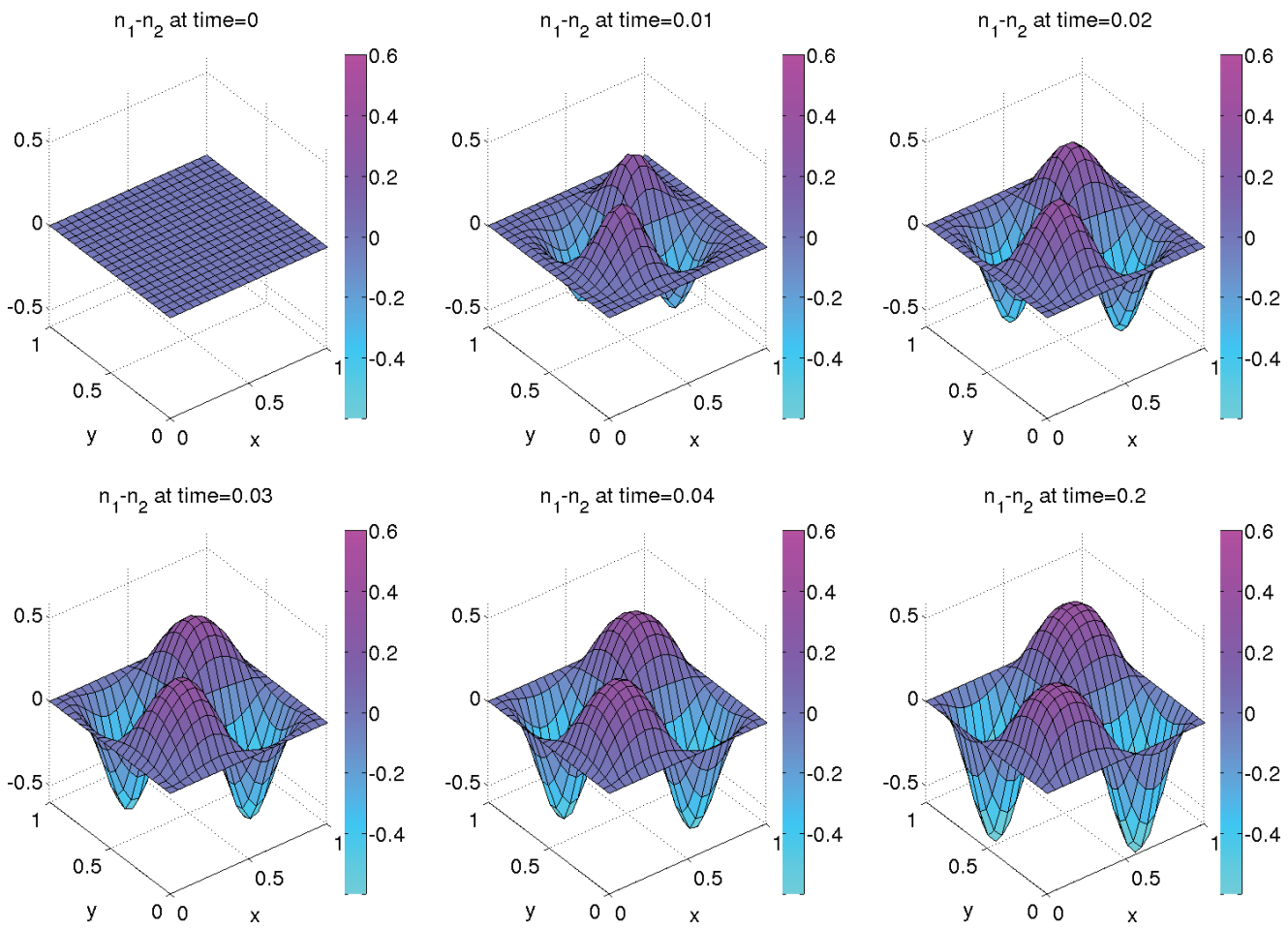

(a) Evolution of the spin polarization for $\varepsilon=0.1$.
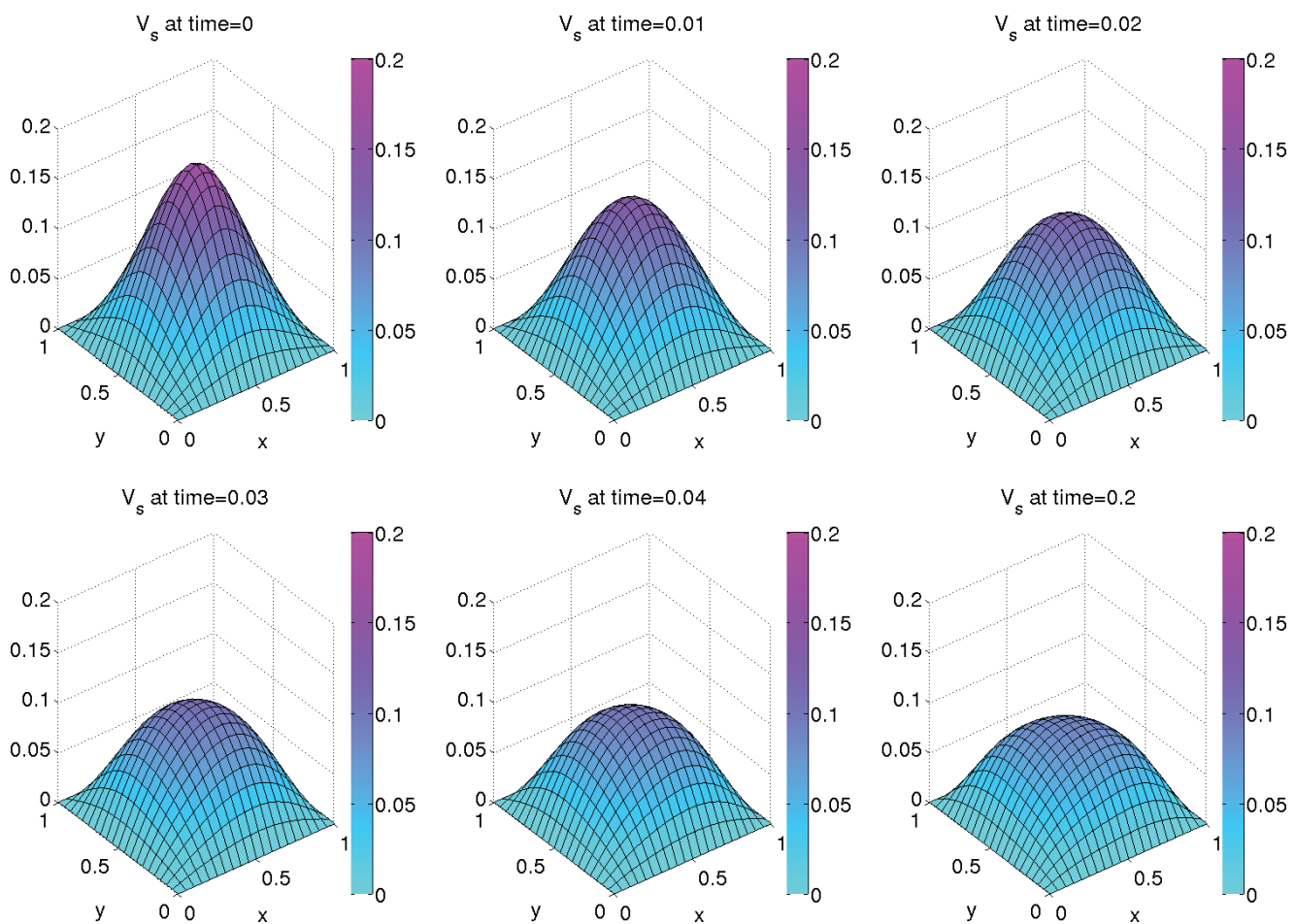

(b) Evolution of the self-consistent electric potential for $\varepsilon=0.1$.

FIG. 5.5. Evolution of the initial state (5.4) towards the steady state 'SP' computed from the stationary Schrödinger-Poisson system depicted in Figure 5.1. Parameters were $\alpha=1.0, \Delta x=\Delta y=$ 0.05 , and $\Delta t=10^{-2}$. The simulation was performed with the semi-implicit scheme 1 . 
has been normalized to a run with the semi-implicit scheme with parameters $\varepsilon=1.0$, $\Delta x=0.1$, and $\Delta t=10^{-2}$. A few remarks concerning Table 5.1:

1. The explicit scheme is subjected to a stability condition with a critical time step $\Delta t_{c}$ for stability that depends on the grid size $\Delta x$ as well as on the semiclassical parameter $\varepsilon$. For all values of $\varepsilon$ and $\Delta x$ studied, it can be seen in Table 5.1 that the time step in the explicit scheme had to be chosen considerably smaller than in the semi-implicit scheme in order to have stability. Moreover, we find that the explicit scheme is unstable for large values of $\varepsilon$, c.f. Remark 4.1. This can be seen from the fact that as $\varepsilon$ passes from 0.1 to 1.0 , for $\Delta x=0.07$, the time step has to be increased by two orders of magnitude to have stability, a typical feature of unstable schemes. Indeed, one observes instabilities when decreasing $\Delta x$ further (and tightening the convergence criterion (5.3); see point 2 . below).

2. For $\varepsilon \geq 0.5$, the time step for the explicit scheme had to be chosen equal to or smaller than the convergence criterion (5.3), thus $\left(\Delta t \leq 10^{-5}\right)$. This manifests itself in the small average of conjugate gradient steps per time step, avg.\#CG, which means that the Functional $\hat{G}_{n}$ might not have been properly minimized at each time step. Indeed, this expected lack of accuracy is seen in the figures 5.6(c) and $5.6(\mathrm{~d})$. Improved accuracy can be achieved by tightening the convergence criterion which in turn leads to considerable increase in computational cost. As mentioned above, for $\varepsilon \approx 1$ and $\Delta x \rightarrow 0$, the explicit scheme is unstable.

3. Regarding CPU-time, the semi-implicit scheme is clearly favorable compared to the explicit scheme for long-time simulations for all parameter values considered.

4. The computational cost depends strongly on $\Delta x$ for both schemes, see footnote 1.

For the parameter sets displayed in Table 5.1, the numerical convergence in $L^{2}$-norm over time of the initial state (5.4) towards the stationary Schrödinger-Poisson states shown in Figure 5.1 is depicted in Figure 5.6. One observes that the explicit scheme, with a time step much smaller than the semi-implicit scheme, converges faster. The achieved accuracy in the steady state is almost identical for the two schemes for $\varepsilon \leq 0.2$ while it is much worse for the explicit scheme for $\varepsilon=1.0$ as discussed in point 2 above. Regarding the semi-implicit scheme, it is obtained that mesh refinement leads to a better accuracy in the steady-state for all $\varepsilon$ considered. Hence, one expects convergence of numerical solutions as $\Delta x \rightarrow 0$, the order of convergence being dependent on the semiclassical parameter $\varepsilon$. Finally, in Figure 5.7, we show numerical convergence towards steady-sate in the case $\varepsilon=0.2$ for the two schemes with different values of $\Delta x$ and the Rashba coupling parameter $\alpha$. Other parameters were chosen as in Table 5.1 for $\varepsilon=0.2$.

\section{Conclusion}

In this work we carried out a numerical investigation of the quantum diffusive spin model introduced in [2] and summarized in equations (2.8)-(2.14). We formally proved (under suitable assumptions) the existence and uniqueness of a solution of two time-discrete versions of this model on the basis of a functional argument. Furthermore, finite difference approximations of space derivatives resulted in two fully discrete schemes which were applied to simulate the evolution of a Rashba electron gas confined in a bounded domain and subjected to spin-dependent external potentials. The first scheme is semi-implicit and advances in time the spin chemical potentials, whereas the second scheme is Euler explicit and advances in time the spin-up and spin-down densi- 

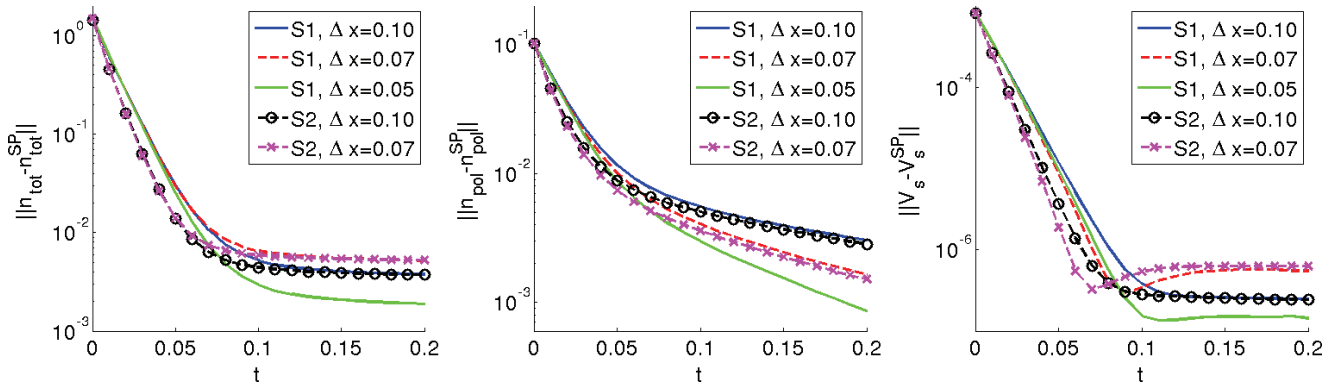

(a) Numerical convergence (in $L^{2}$-norm) for $\varepsilon=0.1$.
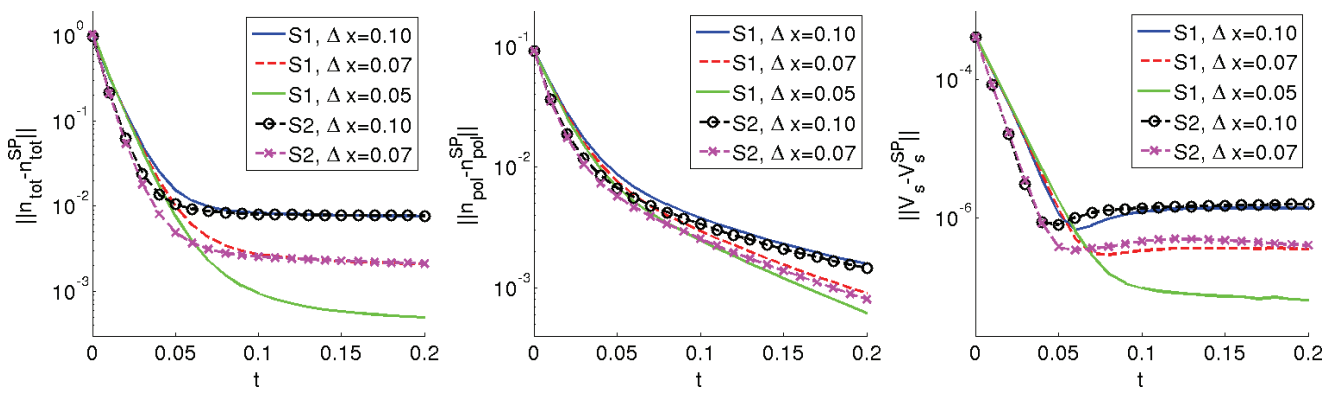

(b) Numerical convergence (in $L^{2}$-norm) for $\varepsilon=0.2$.
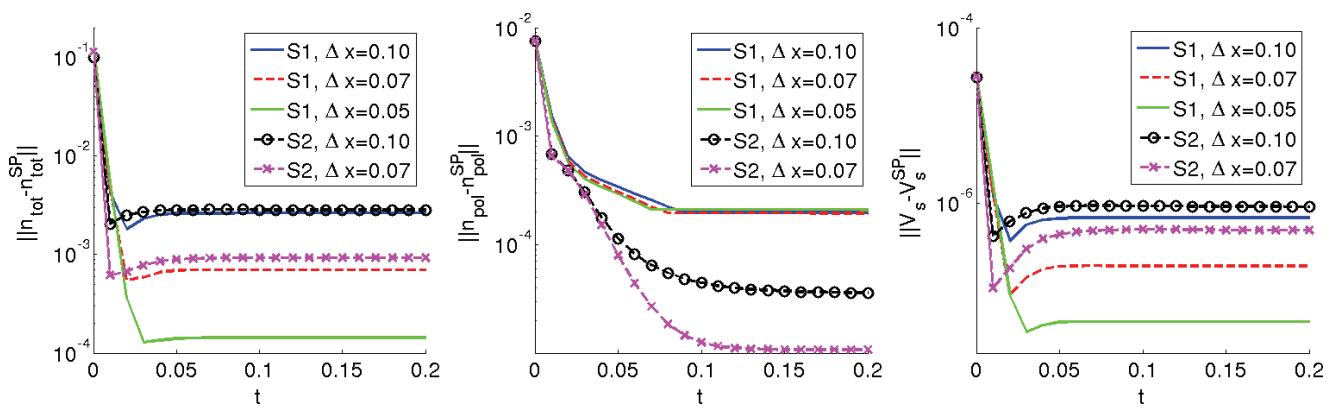

(c) Numerical convergence (in $L^{2}$-norm) for $\varepsilon=0.5$.
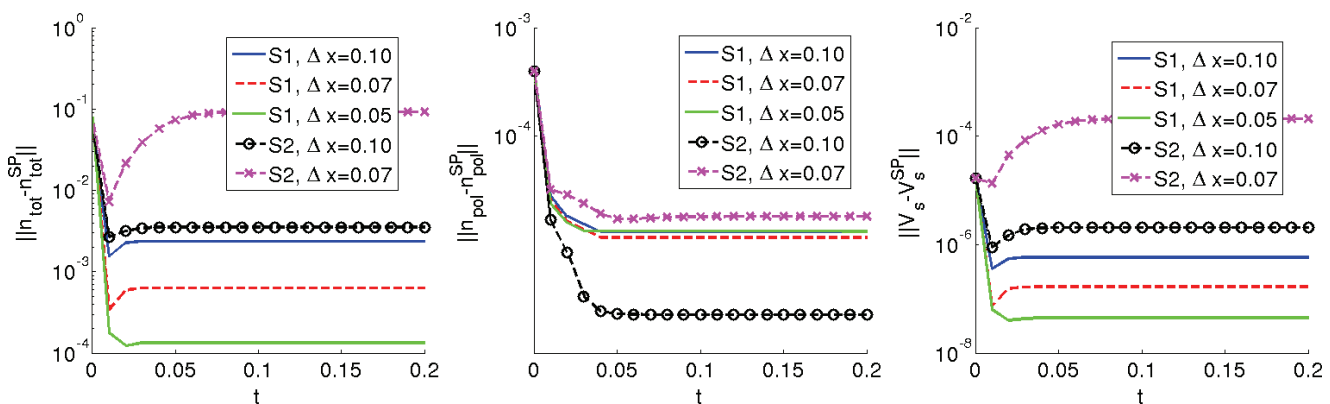

(d) Numerical convergence (in $L^{2}$-norm) for $\varepsilon=1.0$.

FIG. 5.6. Numerical convergence (in $L^{2}$-norm) over time of the initial state (5.4) to the steady states 'SP' (solutions of the stationary Schrödinger-Poisson problem depicted in Figure 5.1) for $\alpha=1.0$ and different values of $\varepsilon$. Results are shown for the semi-implicit scheme (S1) as well as for the explicit scheme (S2) for different space discretizations. The numerical parameters concerning this study can be found in Table 5.1. 

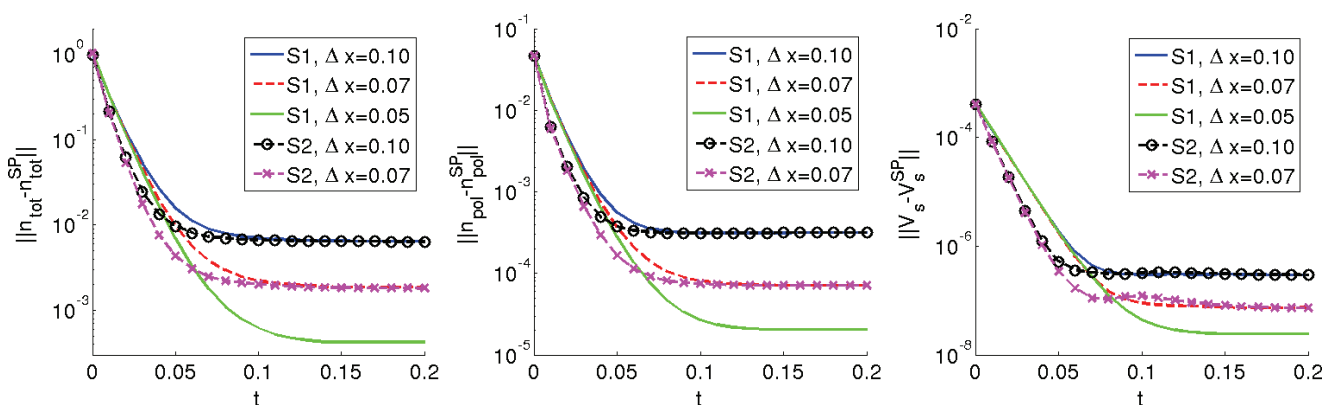

(a) Numerical convergence (in $L^{2}$-norm) for $\alpha=5.0$.
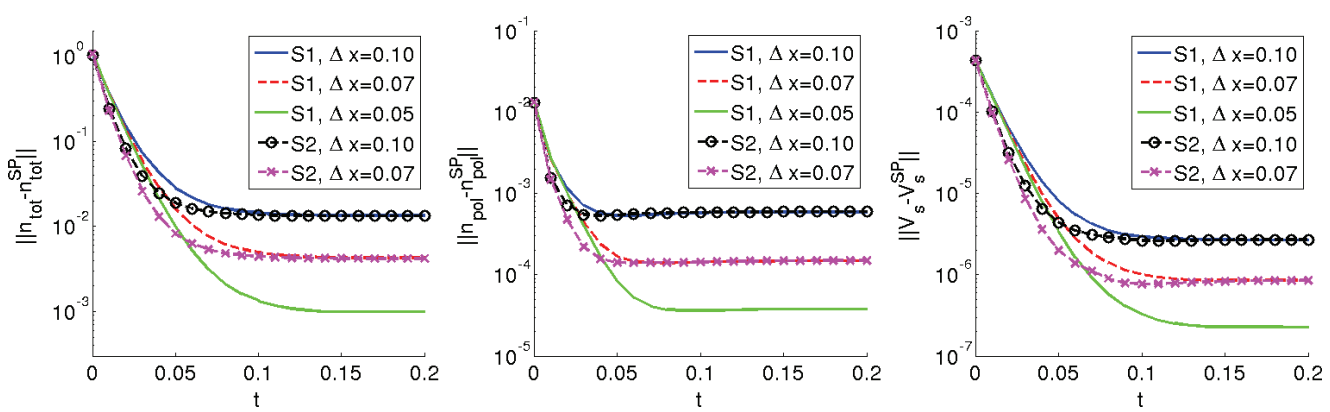

(b) Numerical convergence (in $L^{2}$-norm) for $\alpha=10.0$.
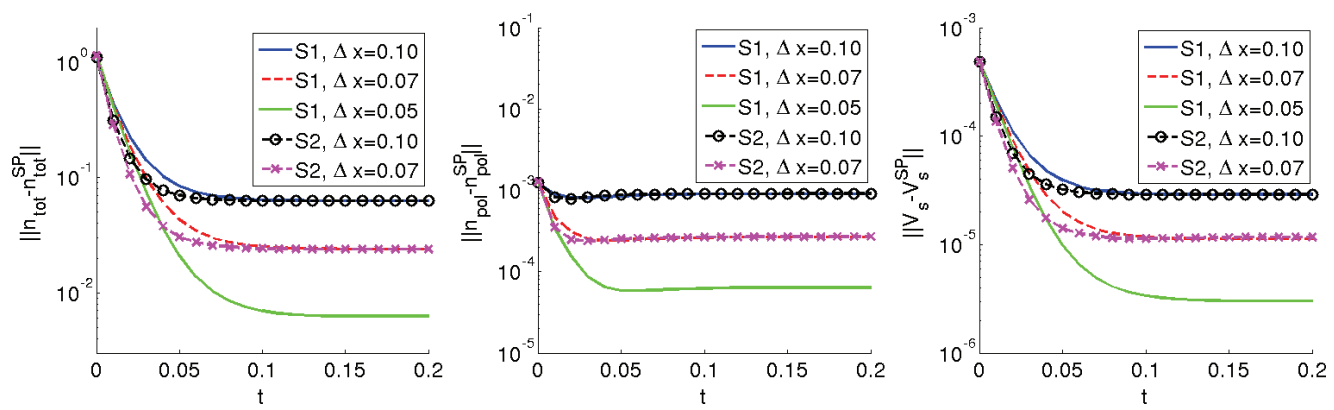

(c) Numerical convergence (in $L^{2}$-norm) for $\alpha=20.0$.

FIG. 5.7. Numerical convergence (in $L^{2}$-norm) over time of the initial state (5.4) to the steady states ' $S P$ ' (solutions of the stationary Schrödinger-Poisson problem depicted in Figure 5.2) for $\varepsilon=0.2$ and different values of $\alpha$. Results are shown for the semi-implicit scheme (S1) as well as for the explicit scheme (S2) for different space discretizations. The numerical parameters concerning this study were the same as those used to obtain Figure 5.4.

ties, respectively. The numerical convergence in the long-time limit of a Gaussian initial condition towards the solution of the corresponding stationary Schrödinger-Poisson problem was demonstrated for different values of the parameters $\varepsilon$ (semiclassical parameter), $\alpha$ (Rashba coupling parameter), $\Delta x$ (grid spacing), and $\Delta t$ (time step). In contrast to the first scheme, the explicit scheme is subjected to a stability condition which makes it less appealing for long-time simulations. Our results show that the quantum drift-diffusion model considered here can be applied for the numerical study of spin-polarized effects due to Rashba spin-orbit coupling and, thus, appears to benefit 
the design of novel spintronics applications.

Appendix A. Perturbed eigenvalue problem. This section is devoted to the computation of the derivatives $\mathrm{d} \lambda_{l}(A)(\delta A)$ and $\mathrm{d} \psi_{l}(A)(\delta A)$ of the eigenvalues and eigenfunctions, respectively, of the Hamiltonian (2.4) when a small perturbation $\delta A$ of the chemical potential $A$ is applied. Let us define

$$
\delta H=\left(\begin{array}{cc}
\delta A_{1} & 0 \\
0 & \delta A_{2}
\end{array}\right)
$$

and start from

$$
(H+\delta H)\left(\psi_{l}+\mathrm{d} \psi_{l}\right)=\left(\lambda_{l}+\mathrm{d} \lambda_{l}\right)\left(\psi_{l}+\mathrm{d} \psi_{l}\right)
$$

where $H$ denotes the Hamiltonian (2.4). Using $H \psi_{l}=\lambda_{l} \psi_{l}$ one obtains, up to first order in the variations,

$$
H \mathrm{~d} \psi_{l}+\delta H \psi_{l}=\lambda_{l} \mathrm{~d} \psi_{l}+\mathrm{d} \lambda_{l} \psi_{l}
$$

Taking now the scalar product with $\psi_{k}$ and using the orthonormalitiy of the eigenfunctions,

$$
\left(\psi_{k}, \psi_{l}\right)_{L^{2}}=\int_{\Omega}\left(\psi_{k}^{1} \overline{\psi_{l}^{1}}+\psi_{k}^{2} \overline{\psi_{l}^{2}}\right) d \mathbf{x}=\delta_{k l}
$$

one obtains

$$
\left(\psi_{k}, H \mathrm{~d} \psi_{l}\right)_{L^{2}}+\left(\psi_{k}, \delta H \psi_{l}\right)_{L^{2}}=\lambda_{l}\left(\psi_{k}, \mathrm{~d} \psi_{l}\right)_{L^{2}}+\mathrm{d} \lambda_{l} \delta_{k l}
$$

Since $H$ is hermitian, we have

$$
\left(\psi_{k}, H \mathrm{~d} \psi_{l}\right)_{L^{2}}=\left(H \psi_{k}, \mathrm{~d} \psi_{l}\right)_{L^{2}}=\lambda_{k}\left(\psi_{k}, \mathrm{~d} \psi_{l}\right)_{L^{2}},
$$

and (A.5) can be written as

$$
\left(\psi_{k}, \delta H \psi_{l}\right)_{L^{2}}=\left(\lambda_{l}-\lambda_{k}\right)\left(\psi_{k}, \mathrm{~d} \psi_{l}\right)_{L^{2}}+\mathrm{d} \lambda_{l} \delta_{k l} .
$$

For $l=k$, we obtain

$$
\mathrm{d} \lambda_{l}(A)(\delta A)=\left(\psi_{l}, \delta H \psi_{l}\right)_{L^{2}}=\int_{\Omega}\left(\left|\psi_{l}^{1}(A)\right|^{2} \delta A_{1}+\left|\psi_{l}^{2}(A)\right|^{2} \delta A_{2}\right) d \mathbf{x}
$$

and for $l \neq k$, assuming that the spectrum of $H$ is non-degenerate, i.e. $\lambda_{l} \neq \lambda_{k}$ for $l \neq k$, one obtains

$$
\left(\psi_{k}, \mathrm{~d} \psi_{l}\right)_{L^{2}}=\frac{\left(\psi_{k}, \delta H \psi_{l}\right)_{L^{2}}}{\lambda_{l}-\lambda_{k}}
$$

Since (A.9) is the projection of $\mathrm{d} \psi_{l}$ onto the $k$-th basis vector of the eigenbasis of $H$ we may write

$$
\mathrm{d} \psi_{l}(A)(\delta A)=\sum_{k \neq l} \frac{\psi_{k}}{\lambda_{l}-\lambda_{k}}\left(\psi_{k}, \delta H \psi_{l}\right)_{L^{2}}
$$




$$
=\sum_{k \neq l} \frac{\psi_{k}(A)}{\lambda_{l}(A)-\lambda_{k}(A)} \int_{\Omega}\left(\psi_{k}^{1}(A) \overline{\psi_{l}^{1}}(A) \delta A_{1}+\psi_{k}^{2}(A) \overline{\psi_{l}^{2}}(A) \delta A_{2}\right) d \mathbf{x} .
$$

Appendix B. The functionals $\mathcal{G}$ and $\mathcal{G}_{n}$. This appendix is concerned with the study of the functionals $\mathcal{G}:\left(H^{1}(\Omega, \mathbb{R})\right)^{2} \rightarrow \mathbb{R}$, introduced in $(3.7)$, and $\mathcal{G}_{n}:\left(H^{1}(\Omega, \mathbb{R})\right)^{2} \rightarrow$ $\mathbb{R}$, introduced in (3.19). The map $\mathcal{G}$ is Gateaux-derivable, and its first and second derivatives in the direction $\delta A=\left(\delta A_{1}, \delta A_{2}\right)$ read

$$
\begin{aligned}
\mathrm{d} \mathcal{G}(A)(\delta A) & =-\sum_{l} e^{-\lambda_{l}(A)} \int_{\Omega}\left(\left|\psi_{l}^{1}(A)\right|^{2} \delta A_{1}+\left|\psi_{l}^{2}(A)\right|^{2} \delta A_{2}\right) d \mathbf{x} \\
\mathrm{d}^{2} \mathcal{G}(A)(\delta A) & =-\sum_{l, k} \frac{e^{-\lambda_{l}}-e^{-\lambda_{k}}}{\lambda_{l}-\lambda_{k}}\left(\int_{\Omega} \psi_{k}^{1} \overline{\psi_{l}^{1}} \delta A_{1} d \mathbf{x}+\int_{\Omega} \psi_{k}^{2} \overline{\psi_{l}^{2}} \delta A_{2} d \mathbf{x}\right)^{2} .
\end{aligned}
$$

Let us present the detailed computation of the second derivative. We have

$$
\begin{aligned}
\mathrm{d}^{2} \mathcal{G}(A)(\delta A)= & -2 \sum_{l} e^{-\lambda_{l}} \int_{\Omega} \mathcal{R e}\left(\overline{\psi_{l}^{1}} \mathrm{~d} \psi_{l}^{1} \delta A_{1}+\overline{\psi_{l}^{2}} \mathrm{~d} \psi_{l}^{2} \delta A_{2}\right) d \mathbf{x} \\
& +\sum_{l} e^{-\lambda_{l}} \mathrm{~d} \lambda_{l} \int_{\Omega}\left(\left|\psi_{l}^{1}\right|^{2} \delta A_{1}+\left|\psi_{l}^{2}\right|^{2} \delta A_{2}\right) d \mathbf{x} .
\end{aligned}
$$

Let us define the following integrals,

$$
I_{1}^{k l}:=\int_{\Omega} \psi_{k}^{1} \overline{\psi_{l}^{1}} \delta A_{1} d \mathbf{x} \quad I_{2}^{k l}:=\int_{\Omega} \psi_{k}^{2} \overline{\psi_{l}^{2}} \delta A_{2} d \mathbf{x} .
$$

Remark that from (A.8) one deduces

$$
\mathrm{d} \lambda_{l}=I_{1}^{l l}+I_{2}^{l l} .
$$

Thus, the second line in (B.1) can be written as

$$
\sum_{l} e^{-\lambda_{l}}\left(I_{1}^{l l}+I_{2}^{l l}\right)^{2}
$$

Moreover, from (A.10) one obtains

$$
\begin{aligned}
\mathrm{d} \psi_{l}^{1} & =\sum_{k \neq l} \frac{\psi_{k}^{1}}{\lambda_{l}-\lambda_{k}}\left(I_{1}^{k l}+I_{2}^{k l}\right), \\
\mathrm{d} \psi_{l}^{2} & =\sum_{k \neq l} \frac{\psi_{k}^{2}}{\lambda_{l}-\lambda_{k}}\left(I_{1}^{k l}+I_{2}^{k l}\right),
\end{aligned}
$$

and therefore we have

$$
\int_{\Omega}\left(\overline{\psi_{l}^{1}} \mathrm{~d} \psi_{l}^{1} \delta A_{1}+\overline{\psi_{l}^{2}} \mathrm{~d} \psi_{l}^{2} \delta A_{2}\right) d \mathbf{x}=\sum_{k \neq l} \frac{1}{\lambda_{l}-\lambda_{k}}\left(I_{1}^{k l}+I_{2}^{k l}\right)^{2} .
$$

The right-hand-side of the first line in (B.1) can now be written as

$$
-2 \sum_{l} \sum_{k \neq l} \frac{e^{-\lambda_{l}}}{\lambda_{l}-\lambda_{k}}\left(I_{1}^{k l}+I_{2}^{k l}\right)^{2}=-\sum_{l, k, l \neq k} \frac{e^{-\lambda_{l}}-e^{-\lambda_{k}}}{\lambda_{l}-\lambda_{k}}\left(I_{1}^{k l}+I_{2}^{k l}\right)^{2} .
$$


Adding (B.4) and (B.7) together and making the convention

$$
l=k: \quad \frac{e^{-\lambda_{l}}-e^{-\lambda_{k}}}{\lambda_{l}-\lambda_{k}}=-e^{-\lambda_{l}},
$$

the second derivative of $\mathcal{G}(A)$ becomes

$$
\mathrm{d}^{2} \mathcal{G}(A)(\delta A)=-\sum_{l, k} \frac{e^{-\lambda_{l}}-e^{-\lambda_{k}}}{\lambda_{l}-\lambda_{k}}\left(I_{1}^{k l}+I_{2}^{k l}\right)^{2} .
$$

As a consequence, the map $\mathcal{G}$ is strictly convex. As far as $\mathcal{G}_{n}$ is concerned, we formally obtain

$$
\begin{aligned}
\mathcal{G}_{n}(A) & =\sum_{l} e^{-\lambda_{l}(A)}+\int_{\Omega} n_{1}^{k} A_{1} d \mathbf{x}+\int_{\Omega} n_{2}^{k} A_{2} d \mathbf{x} \\
& \geq e^{-\lambda_{1}(A)}+\int_{\Omega} n_{1}^{k} A_{1} d \mathbf{x}+\int_{\Omega} n_{2}^{k} A_{2} d \mathbf{x} \underset{\left\|A_{1}\right\|_{L^{2}}+\left\|A_{2}\right\|_{L^{2}} \rightarrow \infty}{\longrightarrow} \infty,
\end{aligned}
$$

where $\lambda_{1}(A)$ stands for the smallest eigenvalue of the Hamiltonian $H(A)$,

$$
\lambda_{1}(A)=\min _{\phi \in\left(H^{1}(\Omega)\right)^{2}}(H(A) \phi, \phi), \quad\|\phi\|_{\left(L^{2}(\Omega)\right)^{2}}=1 .
$$

We are then led to the conclusion that $\mathcal{G}_{n}$ is strictly convex (since its second derivatives coincide with those of $\mathcal{G}$ ) and even coercive.

Appendix C. The functional $\mathcal{F}$. In this appendix, we are concerned with the convexity and coercivity of the functional $\mathcal{F}$, given by (3.9)-(3.12), in order to show that under some assumptions $\mathcal{F}$ admits a unique minimum. The first and second Gateaux derivative of the functionals (3.9)-(3.12) are given by

$$
\begin{aligned}
& \mathrm{d} \mathcal{F}_{1}\left(A, V_{s}\right)\left(\delta A, \delta V_{s}\right)=-\Delta t \int_{\Omega} \nabla \cdot\left(n_{1}^{k} \nabla\left(A_{1}-V_{s}\right)\right)\left(\delta A_{1}-\delta V_{s}\right) d \mathbf{x} \\
& -\Delta t \int_{\Omega} \nabla \cdot\left(n_{2}^{k} \nabla\left(A_{2}-V_{s}\right)\right)\left(\delta A_{2}-\delta V_{s}\right) d \mathbf{x}, \\
& \mathrm{d}^{2} \mathcal{F}_{1}\left(A, V_{s}\right)\left(\delta A, \delta V_{s}\right)=-\Delta t \int_{\Omega} \nabla \cdot\left[n_{1}^{k} \nabla\left(\delta A_{1}-\delta V_{s}\right)\right]\left(\delta A_{1}-\delta V_{s}\right) d \mathbf{x} \\
& -\Delta t \int_{\Omega} \nabla \cdot\left[n_{2}^{k} \nabla\left(\delta A_{2}-\delta V_{s}\right)\right]\left(\delta A_{2}-\delta V_{s}\right) d \mathbf{x} \\
& =\Delta t \int_{\Omega} n_{1}^{k}\left|\nabla\left(\delta A_{1}-\delta V_{s}\right)\right|^{2} d \mathbf{x}+\Delta t \int_{\Omega} n_{2}^{k}\left|\nabla\left(\delta A_{2}-\delta V_{s}\right)\right|^{2} d \mathbf{x}, \\
& \mathrm{d} \mathcal{F}_{2}\left(A, V_{s}\right)\left(\delta A, \delta V_{s}\right)=-\gamma^{2} \int_{\Omega} \Delta V_{s} \delta V_{s} d \mathbf{x}-\int_{\Omega}\left(n_{1}^{k}+n_{2}^{k}\right) \delta V_{s} d \mathbf{x} \\
& +\int_{\Omega} n_{1}^{k} \delta A_{1} d \mathbf{x}+\int_{\Omega} n_{2}^{k} \delta A_{2} d \mathbf{x} \\
& \mathrm{d}^{2} \mathcal{F}_{2}\left(A, V_{s}\right)\left(\delta A, \delta V_{s}\right)=-\gamma^{2} \int_{\Omega}\left(\Delta \delta V_{s}\right) \delta V_{s} d \mathbf{x}=\gamma^{2} \int_{\Omega}\left|\nabla \delta V_{s}\right|^{2} d \mathbf{x},
\end{aligned}
$$




$$
\begin{aligned}
& \mathrm{d} \mathcal{F}_{3}\left(A, V_{s}\right)\left(\delta A, \delta V_{s}\right)=-\alpha \Delta t \mathcal{R e}\left\{\int_{\Omega} \mathcal{D}\left[n_{21}^{k}\left(A_{1}-A_{2}\right)\right]\left(\delta A_{1}+\delta A_{2}-2 \delta V_{s}\right) d \mathbf{x}\right\} \\
&+\alpha \Delta t \mathcal{R e}\left\{\int_{\Omega} n_{21}^{k} \mathcal{D}\left(A_{1}+A_{2}-2 V_{s}\right)\left(\delta A_{1}-\delta A_{2}\right) d \mathbf{x}\right\}, \\
& \mathrm{d}^{2} \mathcal{F}_{3}\left(A, V_{s}\right)\left(\delta A, \delta V_{s}\right)= 2 \alpha \Delta t \mathcal{R e}\left\{\int_{\Omega} n_{21}^{k} \mathcal{D}\left(\delta A_{1}+\delta A_{2}-2 \delta V_{s}\right)\left(\delta A_{1}-\delta A_{2}\right) d \mathbf{x}\right\}, \\
& \mathrm{d} \mathcal{F}_{4}(A)(\delta A)=\frac{2 \alpha \Delta t}{\varepsilon} \mathcal{I} \mathrm{m}\left\{\int_{\Omega}\left(A_{1}-A_{2}\right)\left(\delta A_{1}-\delta A_{2}\right)\left(J_{x}^{21, k}-i J_{y}^{21, k}\right) d \mathbf{x}\right\}, \\
& \mathrm{d}^{2} \mathcal{F}_{4}(A)(\delta A)=\frac{2 \alpha \Delta t}{\varepsilon} \mathcal{I} \mathrm{m}\left\{\int_{\Omega}\left(\delta A_{1}-\delta A_{2}\right)^{2}\left(J_{x}^{21, k}-i J_{y}^{21, k}\right) d \mathbf{x}\right\} .
\end{aligned}
$$

To show that $\mathcal{F}$ is strictly convex, it is sufficient to show that

$$
\mathrm{d}^{2} \mathcal{F}\left(A, V_{s}\right)\left(\delta A, \delta V_{s}\right) \geq 0, \quad \forall \delta A, \delta V_{s} .
$$

One can see immediately that the terms corresponding to $\mathcal{G}, \mathcal{F}_{1}$, and $\mathcal{F}_{2}$ are positive. Nevertheless, nothing can be said about the sign of the terms corresponding to $\mathcal{F}_{3}$ and $\mathcal{F}_{4}$. Assuming, on the other hand, that $\varepsilon$ is a small parameter, which is a physical hypothesis, one can incorporate these latter terms into the former ones. Inspired by a formal proof in [2], we may assume that

$$
n_{21}^{k}=\mathcal{O}\left(\varepsilon^{2}\right), \quad \mathcal{I} \mathrm{m}\left(J_{x}^{21, k}-i J_{y}^{21, k}\right)=2 c \varepsilon \alpha \frac{e^{-A_{1}^{k}}-e^{-A_{2}^{k}}}{A_{2}^{k}-A_{1}^{k}}+\mathcal{O}\left(\varepsilon^{3}\right),
$$

for some constant $c>0$. We remark, then, that the dominant term in $d^{2} \mathcal{F}_{4}$,

$$
4 c \alpha^{2} \Delta t\left\{\int_{\Omega}\left(\delta A_{1}-\delta A_{2}\right)^{2} \frac{e^{-A_{1}^{k}}-e^{-A_{2}^{k}}}{A_{2}^{k}-A_{1}^{k}} d \mathbf{x}\right\},
$$

is positive.

Concerning the coercivity, it is enough to show that

$$
\left|\mathcal{F}\left(A, V_{s}\right)\right| \underset{\|A\|_{H^{1}}+\left\|V_{s}\right\|_{H^{1} \rightarrow \infty}}{\longrightarrow} \infty .
$$

In [8], this property has been shown for the first terms, $\mathcal{X}:=\mathcal{G}+\mathcal{F}_{1}+\mathcal{F}_{2}$, by proving that if $\left|\mathcal{X}\left(A, V_{s}\right)\right|<c_{1}$ for some constant $c_{1}>0$, then there exists a constant $c_{2}>0$ such that $\|A\|_{H^{1}}+\left\|V_{s}\right\|_{H^{1}}<c_{2}$. We can adapt this result in the present case by assuming again that $\varepsilon$ is a small parameter. Indeed, one can again incorporate the new terms $\mathcal{F}_{3}+\mathcal{F}_{4}$ in $\mathcal{X}$ by proving the existence of some constant $C>0$, such that

$$
C\left|\mathcal{X}\left(A, V_{s}\right)\right| \leq\left|\mathcal{F}\left(A, V_{s}\right)\right|,
$$

which proves coercivity. Thus, the functional $\mathcal{F}$, being strictly convex and coercive, admits a unique minimum.

Appendix D. Discretization matrices. Let us present here the discretization matrices used for the fully discrete systems (see Section 4$)$. Let $\mathbb{1}$ stands for the $(N-$ 
$2) \times(N-2)$ identity matrix. Then we have the following discretization matrices:

$$
\begin{aligned}
& D_{x}^{+}=\frac{1}{\Delta x}\left(\begin{array}{cccccc}
-\mathbb{1} & \mathbb{1} & & & \\
0 & -1 & \mathbb{1} & & \\
& \ddots & \ddots & \ddots & \\
& & & 0 & -\mathbb{1} & \mathbb{1} \\
& & & & 0 & 0
\end{array}\right) \in \mathbb{R}^{P \times P}, \\
& D_{x}^{-}=\frac{1}{\Delta x}\left(\begin{array}{ccccc}
0 & 0 & & & \\
-1 & \mathbb{1} & 0 & & \\
& \ddots & \ddots & \ddots & \\
& & -1 & \mathbb{1} & 0 \\
& & & -\mathbb{1} & \mathbb{1}
\end{array}\right) \in \mathbb{R}^{P \times P}, \\
& \begin{array}{c}
D_{y}^{+}=\frac{1}{\Delta y}\left(\begin{array}{ccc}
d_{y}^{+} & & \\
& \ddots & \\
& & d_{y}^{+}
\end{array}\right), \quad D_{y}^{-}=\frac{1}{\Delta y}\left(\begin{array}{ccc}
d_{y}^{-} & & \\
& \ddots & \\
& & d_{y}^{+}, D_{y}^{-} \in \mathbb{R}^{P \times P}
\end{array}\right),
\end{array} \\
& d_{y}^{+}=\left(\begin{array}{cccccc}
-1 & 1 & & & \\
0 & -1 & 1 & & \\
& & \ddots & \ddots & \ddots & \\
& & & 0 & -1 & 1 \\
& & & & 0 & 0
\end{array}\right) \in \mathbb{R}^{(N-2) \times(N-2)},
\end{aligned}
$$

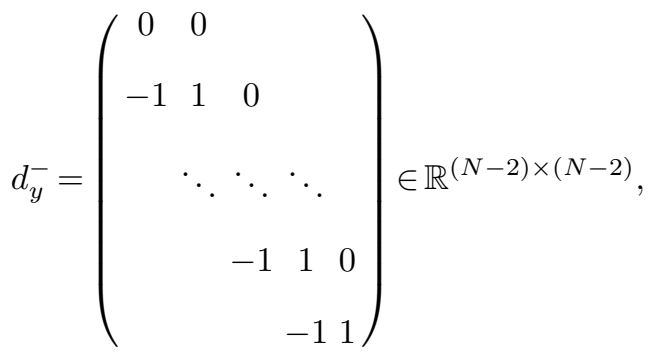

$$
\begin{aligned}
& \widetilde{D}_{x}=\frac{D_{x}^{+}+D_{x}^{-}}{2}, \quad \widetilde{D}_{y}=\frac{D_{y}^{+}+D_{y}^{-}}{2}, \\
& \Delta_{d i r}=\Delta^{x}+\Delta^{y} \quad \in \mathbb{R}^{P \times P},
\end{aligned}
$$




$$
\begin{aligned}
& \Delta^{x}=\frac{1}{(\Delta x)^{2}}\left(\begin{array}{ccccc}
-2 \mathbb{1} & \mathbb{1} & & & \\
\mathbb{1} & -2 \mathbb{1} & \mathbb{1} & & \\
& \ddots & \ddots & \ddots & \\
& & \mathbb{1} & -2 \mathbb{1} & \mathbb{1} \\
& & & \mathbb{1} & -2 \mathbb{1}
\end{array}\right) \in \mathbb{R}^{P \times P}, \\
& \Delta^{y}:=\frac{1}{(\Delta y)^{2}}\left(\begin{array}{ccc}
l_{y} & & \\
& \ddots & \\
& & \\
& & l_{y}
\end{array}\right) \in \mathbb{R}^{P \times P} \\
& l_{y}=\left(\begin{array}{cccccc}
-2 & 1 & & & \\
1 & -2 & 1 & & \\
& & \ddots & \ddots & \ddots & \\
& & & 1 & -2 & 1 \\
& & & & 1 & -2
\end{array}\right) \in \mathbb{R}^{(N-2) \times(N-2)},
\end{aligned}
$$

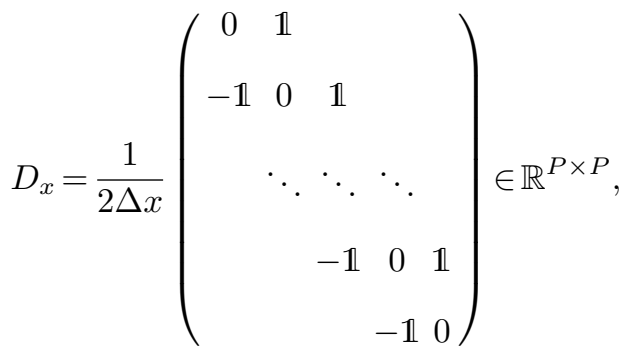

$$
\begin{aligned}
& D_{y}:=\frac{1}{2 \Delta y}\left(\begin{array}{ccc}
d_{y} & & \\
& \ddots & \\
& & \\
& & d_{y}
\end{array}\right) \in \mathbb{R}^{P \times P},
\end{aligned}
$$

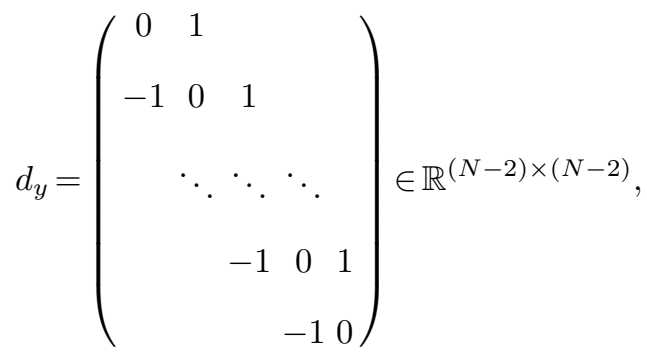




$$
\begin{aligned}
& D_{x}^{b}=\frac{1}{\Delta x}\left(\begin{array}{ccccc}
\mathbb{1} & 0 & & & \\
-\mathbb{1} & \mathbb{1} & 0 & & \\
& \ddots & \ddots & \ddots & \\
& & -\mathbb{1} & \mathbb{1} & 0 \\
& & & -\mathbb{1} & \mathbb{1}
\end{array}\right) \in \mathbb{R}^{P \times P}, \\
& D_{y}^{b}=\frac{1}{\Delta y}\left(\begin{array}{ccc}
d_{y}^{b} & & \\
& \ddots & \\
& & d_{y}^{b}
\end{array}\right) \in \mathbb{R}^{P \times P}, \\
& d_{y}^{b}=\left(\begin{array}{ccccc}
1 & 0 & & & \\
-1 & 1 & 0 & & \\
& \ddots & \ddots & \ddots & \\
& & -1 & 1 & 0 \\
& & & & \\
& & & & 1
\end{array}\right) \in \mathbb{R}^{(N-2) \times(N-2)} .
\end{aligned}
$$

Acknowledgments. This work has been supported by the ANR project QUATRAIN and by the French-Italian research project GREFI-MEFI. S. Possanner acknowledges the support from the Austrian Science Fund, Vienna, under the contract number P21326-N16. L. Barletti acknowledges support from the Italian National Project (PRIN) Mathematical problems of kinetic theories and applications, prot. 2009NAPTJF 003.

\section{REFERENCES}

[1] M. Ancona, Diffusion-drift modeling of strong inversion layers, COMPEL, 6, 11-18, 1987.

[2] L. Barletti and F. Méhats, Quantum drift-diffusion modeling of spin transport in nanostructures, J. Math. Phys., 51, 053304(20), 2010.

[3] Y. Bychkov and E.I. Rashba, Properties of a 2D electron gas with lifted spectral degeneracy, JETP Letters, 39(2), 78-81, 1984.

[4] P. Degond, S. Gallego, and F. Méhats, An entropic quantum drift-diffusion model for electron transport in resonant tunneling diodes, J. Comput. Phys., 221, 226-249, 2007.

[5] P. Degond, F. Méhats, and C. Ringhofer, Quantum energy-transport and drift-diffusion models, J. Stat. Phys., 118(3-4), 625-667, 2005.

[6] P. Degond and C. Ringhofer, Quantum moment hydrodynamics and the entropy principle, J. Stat. Phys., 112(3-4), 587-628, 2003.

[7] B. Derrida, J. Lebowitz, E. Speer, and H. Spohn, Fluctuations of a stationary nonequilibrium interface, Phys. Rev. Letters, 67, 165-168, 1991.

[8] S. Gallego and F. Méhats, Entropic discretization of a quantum drift-diffusion model, SIAM J. Numer. Anal., 43(5), 1828-1849, 2006.

[9] R. El Hajj, Diffusion models for spin transport derived from the spinor Boltzmann equation, Commun. Math. Sci., 12(13), 565-592, 2014.

[10] A. Jüngel, Transport Equations for Semiconductors, Springer, Berlin, 2009.

[11] R. LeVeque, Numerical Methods for Conservation Laws, Second Edition, Birkhäuser, BaselBoston-Berlin, 1992.

[12] P. Markowich, C. Ringhofer, and C. Schmeiser, Semiconductor Equations, Springer, Vienna, 1990. 
[13] F. Méhats and O. Pinaud, An inverse problem in quantum statistical physics, J. Stat. Phys., 140, 565-602, 2010.

[14] F. Méhats and O. Pinaud, A problem of moment realizability in quantum statistical physics, Kinet. Relat. Models, 4(4), 1143-1158, 2011.

[15] F. Nier, A stationary Schrödinger-Poisson system arising from the modelling of electronic devices, Forum Math., 2, 489-510, 1990.

[16] F. Nier, A variational formulation of Schrödinger-Poisson systems in dimension $d \leq 3$, Commun. Part. Diff. Eqs., 18, 1125-1147, 1993.

[17] S. Possanner and C. Negulescu, Diffusion limit of a generalized matrix Boltzmann equation for spin-polarized transport, Kinet. Relat. Models, 4(4), 1159-1191, 2011.

[18] F. Poupaud, Diffusion approximation of the linear semiconductor Boltzmann equation: analysis of boundary layers, Asympt. Anal., 4, 293-317, 1991.

[19] W. van Roosbroeck, Theory of flow of electrons and holes in germanium and other semiconductors, Bell Syst. Techn. J., 29, 560-607, 1950.

[20] S. Saikin, Drift-diffusion model for spin-polarized transport in a non-degenerate 2DEG controlled by spin-orbit interaction, J. Phys.: Condens. Matter, 16, 5071-5081, 2004.

[21] I. Žutić, J. Fabian, and S. Das Sarma, Spintronics: fundamentals and applications, Rev. Mod. Phys., 76(2), 323-410, 2002. 